\title{
Optimal Control and Necessary Optimality Conditions for Nonlinear and Perturbed Dynamic Problems
}

\author{
Gossan D. Pascal Gershom ${ }^{1}$, Bailly Balé ${ }^{2} \&$ Yoro Gozo $^{1}$ \\ ${ }^{1}$ University Nangui Abrogoua, UFR-SFA, department of Mathematics, Côte d'Ivoire \\ ${ }^{2}$ University F.H.B, UFR in Mathematics and Computer Science, Côte d'Ivoire \\ Correspondence: Gossan D. Pascal Gershom, University Nangui Abrogoua, UFR-SFA, department of Mathematics, 01 \\ BP 5670 Abidjan 01, Côte d'Ivoire. E-mail: gershom2011@yahoo.fr
}

Received: September 16, 2018 Accepted: October 17, 2018 Online Published: November 12, 2018

doi:10.5539/jmr.v10n6p63

URL: https://doi.org/10.5539/jmr.v10n6p63

\begin{abstract}
The main goal of this paper is to establish the first order necessary optimality conditions for a tumor growth model that evolves due to cancer cell proliferation. The phenomenon is modeled by a system of three-dimensional partial differential equations. We prove the existence and uniqueness of optimal control and necessary conditions of optimality are established by using the variational formulation.
\end{abstract}

Keywords: nonlinear system, optimal control, existence and uniqueness, first-order necessary optimality condition

\section{Introduction}

Mathematical models are increasingly used, particularly in medicine. Formalising biological phenomena such as tumours (Robiyn, 2004), which is the subject of our research, is a hot topic both elsewhere and in Côte d'Ivoire. The first mathematical models of tumour growth that we know of date back to the 1930. However, it was essentially towards the end of the 20th century that many of them were developed (William, 1932). Among the different models, a distinction is usually made between discrete and continuous models. In a discrete model, each entity is represented individually and reacts to given biophysical rules. Biological processes, such as progression in the cell cycle, can then be translated in detail. Moreover, the fate of all entities can be known, which facilitates comparisons between the mathematical model and the experimental data. However, these advantages reach their limits in large cellular populations: it then becomes necessary to monitor many entities, which proves costly in terms of numerical resolution. The use of continuous models makes it possible to overcome this disadvantage and to model large populations. Indeed, in a continuous model, elements are described in terms of population density and their actions are modelled by partial differential equations: this is an advantage for studying the mathematical properties of the model, but it makes it difficult to establish direct links between model parameters and physical measurements. In this paper we propose the use of optimal control theory (Fursikov, 2000) and (Sritharan, 1998) to provide a complete explanation of biological phenomena. Optimal Control Theory is the contemporary framework for analyzing and solving optimization problems, born in the 1960s with the work of (Pontryagin and al, 1962) based on earlier contributions by (Lagrange, 1788) and (Hamilton, 1827). Essentially, optimal control theory considers the problem of how to achieve an objective subject to external constraints, and it has mainly been used in economics. To our knowledge, in the biosciences, optimal control theory has been applied to the design of optimal therapies, optimal harvesting policies and optimal investments in renewable resources, but not to the origin of observed biological behaviours. When designing an optimal therapy, optimal harvest or optimal investment, the goal is to achieve an objective external to the biological entities involved, namely: minimize (Raymond, 2013) the negative effects of drugs and diseases and maximize the current value of revenues, subject to biological laws describing existing effects. The appropriate mathematical approach to this problem is therefore the optimal control theory. However, in addition to these well-known applications, optimal control theory is also the most appropriate approach for studying biological phenomena understood as the result of the behaviour of semi-autonomous bio-entities. Therefore, optimal control theory provides a comprehensive explanation of observed behaviors: bio-entities pursue their own specific goals, the actions of one bioentity affect the ability of other entities to achieve their goals, and therefore, all behaviors are interdependent. However, the interpretation of biological phenomena as a result of a set of optimal control problems has not yet been considered by current biomathematics. In this respect, using non-linear dynamic models as a starting point, the aim of this paper is to show how this application of optimal control theory is a promising approach for the analysis of biomedical questions, i.e. to establish the necessary optimality conditions on a dynamic system on which one can act by means of a command to go from a given initial state to a very precise final state. One of the necessary (attractive) aspects of the order is to introduce a functional taking into account the entire trajectory of the system up to a final horizon. The objective will therefore be to determine a control that makes it possible to manipulate the system according to its dynamics while minimizing the cost 
function, that is to say, determine a solution having an optimal quality.

The rest of the paper is organized as follows: after this introduction, in section 2 we describe the mathematical model that we will study and present some functional spaces, then using continuous linear operators of Nemytskii and Hammerstein, we get the linearization of the problem with some established assumptions. In section 3, we formulate the optimal control problem and prove the existence and uniqueness of an optimal solution for the controlled system with functional cost. In Section 4, we establish the functional gradient and formulate the adjoint problem of the initial problem and finally the establishment of the necessary conditions of optimality of the first order associated with the problem are studied in section 5.

\section{Mathematical Models for Tumour Dynamics}

Tumour dynamics modelling is an active research area for biologists, mathematicians and engineers. Different approaches are used in mathematical modelling of cancer and its control.(Swanson, 2000) models multiform tumor (malignant brain tumor) using partial differential equations. Some researchers have also studied the tumour growth model using cellular automata that may include very specific characteristics of the tumor, patient and effective drug in model (Kansal, 2000);(Gerlee, 2007).(Anderson \& Chaplain, 1998) and (Anderson \& Enderling, 2006) also used the approach of cellular automata to model tumour growth, angiogenesis and metastasis. Another different approach is the work of (Pillis \& Radunskaya, 2003) in which they construct a general tumor growth model, using ordinary differential equations, which show the dynamics of tumor growth using the number of healthy cells and immune cells. In this paper, we present a model based on the one presented in (Gossan, Yoro \& Bally, 2018) of non-linear differential reaction-diffusion equations, describing the proliferative evolution of tumor cells across a given domain.

Let us designate by $x$ the size of the tumor, and $t$ the time parameter. Consider a time-dependent reference region $I_{t}=I \times(0, T), T>0$ occupied by the tumor where $I$ is a bounded open set of $\mathbb{R}^{3}$ and either $\partial I_{t}=\partial I \times(0, T)$, its pretty smooth border. Let us $\overrightarrow{\mathbf{n}}$ as the unit outward normal to the boundary $\partial I$.

Note $v=v(x, t)$ and $\xi_{e}=\xi_{e}(x, t)$, vector functions designating respectively the proliferation rate of cancer cells, the density of external forces ( healthy cells + nutrients + constant drug supply) and the scalar function $\rho=\rho(x, t)$, the volume density of tumor cells. The model is then described by the following equations:

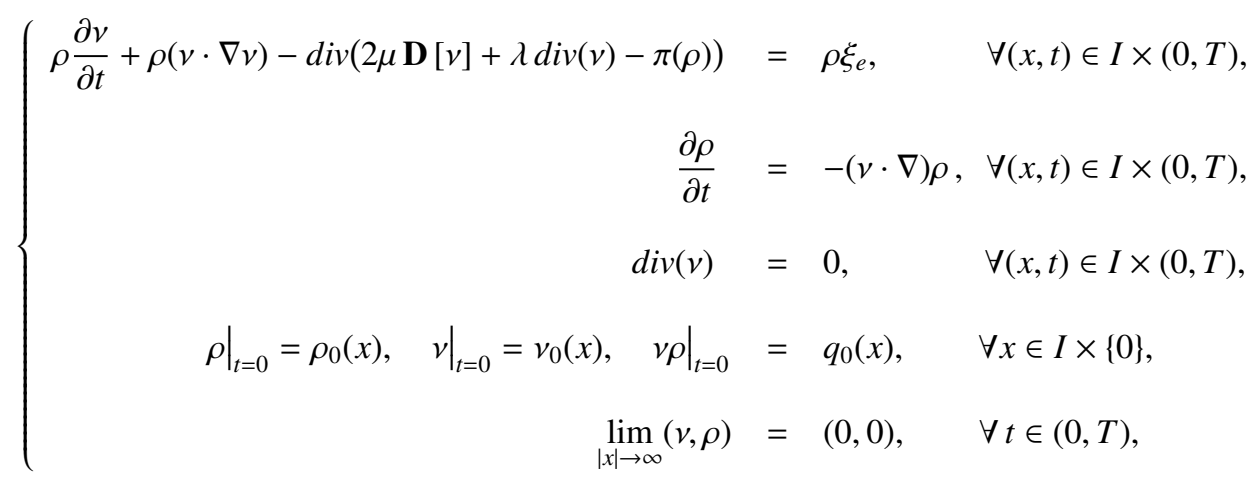

where $\mathbf{D}[v]=\frac{1}{2}\left(\frac{\partial v_{j}}{\partial x_{i}}+\frac{\partial v_{i}}{\partial x_{j}}\right)$ (for $1 \leq i, j \leq 3$ ) is the deformation rate tensor.

The system (2.1) is represented by the nonlinear differential equations, whose equation $(2.1)_{1}$, called the continuity equation, reflects the principle of mass conservation. The function $(v \cdot \nabla) \rho$ is the moment of the cells, while the equation $(2.1)_{2}$, called the quantity of motion equation, is derived from a combination of the fundamental principle of the dynamics of the equation $(2.1)_{1}$, and $(2.1)_{3}$ translated the incompressibility of the system.

It consists of a diffusion term $\operatorname{div}(2 \mu \mathbf{D}[v]+\lambda \operatorname{div}(v))$ with viscosity coefficients $\mu$ and $\lambda$, and a tumor cell convection term $(v \cdot \nabla v)$ is given by $\sum_{i=1}^{3} v_{i} \partial_{x_{i}} v$.

We assume that on the $I$ boundary of the domain, the velocity verifies :

$$
\left.v\right|_{\partial I}=0, \quad \forall(x, t) \in \partial I \times(0, T) .
$$

For physical reasons, $\mu$ and $\lambda$ meet the following conditions :

$$
\mu>0, \quad 2 \mu+\lambda>0 .
$$

It thus appears in the equation of conservation of the momentum two diffusion terms modeling the effects of small scales. Indeed, the viscosity reflects the friction forces at the microscopic level. To get an idea, one could imagine such forces as 
those that force a liquid to flow slowly. The pressure $\pi$ depends on the variable density, and is given by the following state law :

$$
\pi=\kappa \rho^{\alpha}, \quad \kappa \geq 1,
$$

and $\alpha$ the adiabatic constant is such that $\alpha>(d-1) / 2(d=3)$.

Before announcing the results, it is necessary to define the areas in which we are working. In this sub-section, we introduce the notation that will be used throughout this document.

\subsection{Notations and Functional Framework}

In this work, a couple of symbols and definitions are used, which are generally introduced when they are needed. However, some general notations that belong to the mathematical norm are given here for reference in advance. The following function spaces provide a norm framework for studying optimal conditions of problem. (2.1) - (2.2).

The underlying domain. Let $I \subset \mathbb{R}^{3}$, a delimited domain $\partial I$ its sufficiently smooth border. For $T>0$, the interval $(0, T)$ defines the considered time interval and $I_{t}=I \times(0, T)$ a space-time domain with boundary $\partial I_{t}=\partial I \times(0, T)$.

Standard operators. $x=\left(x_{1}, x_{2}, x_{3}\right)$ is the space variable in $\mathbb{R}^{3}$. For $x, y \in \mathbb{R}^{3}, x \cdot y=\sum_{i=1}^{3} x_{i} y_{i} . \nabla$ is the gradient ans $\Delta$ is the laplacian. When $\mathscr{G}(x)=\left(\mathscr{G}_{1}, \mathscr{G}_{2}, \mathscr{G}_{3}\right)$ is an $\mathbb{R}^{3}$-valued function,

$$
\begin{gathered}
\nabla \cdot \mathscr{G}=\sum_{i=1}^{3} \frac{\partial \mathscr{G}_{i}}{\partial x_{i}}, \quad|\nabla \mathscr{G}|^{2}=\sum_{i, j=1}^{3}\left|\frac{\partial \mathscr{G}_{i}}{\partial x_{j}}\right|^{2}, \quad\|\mathscr{G}\|_{L^{p}\left(I ; \mathbb{R}^{3}\right)}=\left(\sum_{i=1}^{3}\left\|\mathscr{G}_{i}\right\|_{L^{p}\left(I ; \mathbb{R}^{3}\right)}^{p}\right)^{1 / p}, \\
\|\nabla \mathscr{G}\|_{L^{p}\left(I ; \mathbb{R}^{3}\right)}=\left(\sum_{i, j=1}^{3}\left\|\frac{\partial \mathscr{G}_{i}}{\partial x_{j}}\right\|_{L^{p}\left(I ; \mathbb{R}^{3}\right)}^{p}\right)^{1 / p} .
\end{gathered}
$$

Standard Lebesgue spaces. Let $m$ be a non-negative integer. We denote by $H^{m}\left(I ; \mathbb{R}^{3}\right)$ the usual Sobolev space $W^{m, 2}\left(I ; \mathbb{R}^{3}\right)$ as defined in (Lions \& Magenes, 1972).

We note by $\mathcal{D}(I)$, the space of infinitely differentiable functions with compact support. Its closing in the norm $W^{m, p}\left(I ; \mathbb{R}^{3}\right)$ $(2 \leq p<s<+\infty)$ is noted by $W_{0}^{m, p}\left(I ; \mathbb{R}^{3}\right)$. An alternate characteristic in the case where $m=1$ and $p=2$ is

$$
\operatorname{ker} \gamma_{0}=W_{0}^{1,2}\left(I ; \mathbb{R}^{3}\right)=\left\{v \in H^{1}\left(I ; \mathbb{R}^{3}\right): \gamma_{0} v=0\right\},
$$

where $\gamma_{0}$ is the $v$ trace operator. We also note by $L^{p}(I)^{3}=L^{p}\left(I ; \mathbb{R}^{3}\right)$, the lebesgue space on $I$ provided with the norm $\|.\|_{p}$ and by $\|.\|_{\mathbb{E}}$ the norm associated with a space $\mathbb{E}$. If $\mathbb{E}$ is a Banach space, $L^{p}(0, T ; \mathbb{E})$ is the Banach space composed of functions, measurable on $(0, T)$ which value in $\mathbb{E}$. For details concerning these spaces, see (Adams, 1945) or (Girault ,1986). We consider zero divergence spaces introduced for the problem $(2.1)-(2.2)$.

$$
\begin{aligned}
C_{0, \sigma}^{\infty}\left(I ; \mathbb{R}^{3}\right) & :=\left\{v \in \mathcal{D}\left(I ; \mathbb{R}^{3}\right): \operatorname{div}(v)=0\right\}, \\
\mathbb{K}_{d i v}^{1} & :=\left\{v \in L^{2}\left(I ; \mathbb{R}^{3}\right): \operatorname{div}(v)=0,\left.v \cdot \mathbf{n}\right|_{\partial I}=0\right\}, \\
\mathbb{K}_{d i v}^{0} & :=\left\{v \in W_{0}^{1,2}\left(I ; \mathbb{R}^{3}\right): \operatorname{div}(v)=0\right\},
\end{aligned}
$$

where $\mathbb{K}_{d i v}^{0}$ and $\mathbb{K}_{d i v}^{1}$ the respective closure of $C_{0, \sigma}^{\infty}\left(I ; \mathbb{R}^{3}\right)$ in $W_{0}^{1,2}\left(I ; \mathbb{R}^{3}\right)$ and $L^{2}\left(I ; \mathbb{R}^{3}\right)$.

Note by $\mathbb{X}_{v}^{0}=L^{\infty}\left((0, T) ; \mathbb{K}_{d i v}^{1}\right)$, and $\mathbb{X}_{\xi_{e}}^{0}=L^{1}\left((0, T) ; L^{\frac{2 s}{s-1}}\left(I ; \mathbb{R}^{3}\right)\right)$, spaces of the continuous functions of integrable square. These are banach spaces for the respective norms $\|v\|_{\mathbb{X}_{v}^{0}} \stackrel{\text { def }}{=}\left(\int_{0}^{T}\|v\|_{\mathbb{K}_{d i v}^{1}}^{2} d t\right)^{\frac{1}{2}}$ obtained by the closing the functions of $C^{2}(I \times(0, T))$ in metric $L^{\infty}\left((0, T) ; \mathbb{K}_{d i v}^{1}\right)$.

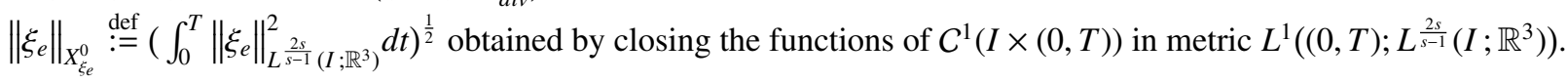

\subsection{Linearization of the Problem}

The characteristics are defined as previously. That is to say, we consider a bounded domain $I$ with the same initial conditions. In this paragraph, we construct a linear functional perturbation that linearizes the $(2.1)_{1}$ equation and we give the characteristics of the functions that compose it. However, let's look at the term $(v \cdot \nabla v)$ that appears in the equation $(2.1)_{1}$. It is at the root of the difficulties encountered in solving this problem. We will therefore linearize the system by substituting this term by the following perturbation :

$$
\mathfrak{F}(\mathcal{H}, \varphi) \stackrel{\text { def }}{=} \mathcal{H}_{p}(x, t)+\partial_{v} \varphi(x, t, v, w)
$$


$\varphi$ is a measurable function following $(x, t)$, twice continuously differentiable with respect to $(v, w) \in \mathbb{R}^{3} \times \mathbb{R}^{9}$, and $\mathcal{H}_{p}=\mathcal{P} \vartheta$ a continuous integral operator (see Silvia, 2014) which, at any function $\vartheta$, matches $\mathcal{H}_{p}$. That is written in expanded form :

$$
\mathcal{H}_{\mathcal{P}} \vartheta(., t): \stackrel{\text { def }}{=} \int_{0}^{T} \int_{I} \mathcal{P}\left(x-y, t-t^{\prime}\right) \partial_{v} \vartheta\left(y, t^{\prime}, v, w\right) d y d t, \quad t>t^{\prime}, \quad \forall x, y \in I,
$$

where the $\mathcal{P}\left(x-y, t-t^{\prime}\right)$ operator is a linear and continuous application in $I \times(0, T)$. Using the new functions introduced, the initial value problem (2.1) is reformulated as follows

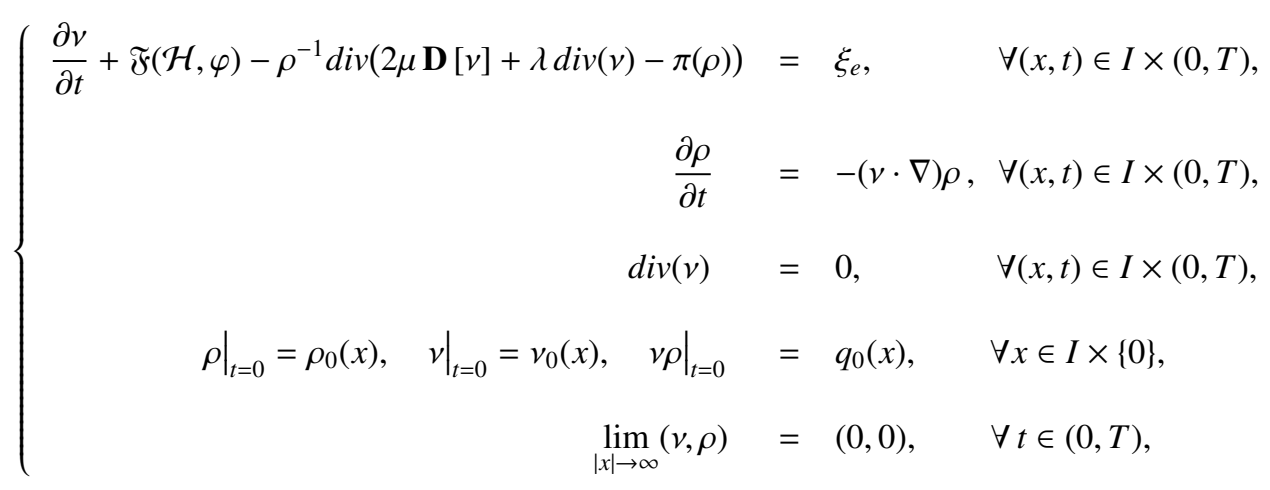

Note that this system is a simpler version of the (2.1) system since the term $(v \cdot \nabla v)$ has been replaced by $\mathfrak{F}(\mathcal{H}, \varphi)$. This approach introduced new variables $v, w$ which are considered respectively as an argument of the $v(x, t)$ field and its divergence. We will then make some hypotheses about the functions $\varphi(x, t, v, w)$ and $\vartheta\left(y, t^{\prime}, v, w\right)$, defined on $I \times(0, T) \times \mathbb{R}^{3} \times \mathbb{R}^{9} \longrightarrow \mathbb{R}^{9}$, and then give the definition of the general solution of the problem $(2.7)_{1}-(2.7)_{5}$, subject to the limit condition (2.2).

\subsubsection{Assumptions and Definition}

(H-1): Let $\beta, \widetilde{\beta}>0$ and $T>0$ (fixed). For every $(v, w) \in \mathbb{R}^{3} \times \mathbb{R}^{9}$, the functions $(x, t, v, w) \longmapsto \varphi(x, t, v, w)$ and $\left(y, t^{\prime}, v, w\right) \longmapsto \vartheta\left(y, t^{\prime}, v, w\right)$ are measurable and verify the following conditions :

$$
\begin{aligned}
& |\varphi(x, t, v, w)| \leq \beta^{-1}\left(|v|^{2}+|w|^{2}\right) \exp (T), \\
& \left|\vartheta\left(y, t^{\prime}, v, w\right)\right| \leq \widetilde{\beta}^{-1}\left(|v|^{2}+|w|^{2}\right) \exp (T) .
\end{aligned}
$$

(H-2) : For almost every $(x, t),\left(y, t^{\prime}\right) \in I \times(0, T)$, the functions $(x, t, v, w) \longmapsto \varphi(x, t, v, w)$ and $\left(y, t^{\prime}, v, w\right) \longmapsto \vartheta\left(y, t^{\prime}, v, w\right)$ are twice continuously differentiable with respect to couples $(v, w)$. Morever : $\forall(v, w) \in \mathbb{R}^{3} \times \mathbb{R}^{9}$

$$
\begin{aligned}
& \left|\Delta_{v} \varphi\right|+\left|\Delta_{w} \varphi\right| \leq 4 \beta^{-1} \exp (T), \\
& \left|\Delta_{v} \vartheta\right|+\left|\Delta_{w} \vartheta\right| \leq 4 \widetilde{\beta}^{-1} \exp (T) .
\end{aligned}
$$

(H-3) : Let $\mathcal{A}_{\epsilon}$ and $\mathcal{B}_{\epsilon}$ be two nonlinear $F$-differentiable and $G$-differentiable operators. We note by $\mathcal{A}_{\epsilon}^{\prime \prime}$ and $\mathcal{B}_{\epsilon}^{\prime \prime}$, the respective second differential of $\mathcal{A}_{\epsilon}$ and $\mathcal{B}_{\epsilon}$ defined as follows

$$
\begin{aligned}
\mathcal{A}_{\epsilon}^{\prime \prime}: L^{\infty}\left((0, T) ; \mathbb{K}_{d i v}^{1}\right) & \longrightarrow \mathcal{L}_{2}\left(\mathbb{X}_{v}^{0}, L^{2}\left((0, T) ; L^{2}\left(I ; \mathbb{R}^{3}\right)\right)\right) \\
v(x, t) & \longmapsto \mathcal{A}_{\epsilon}^{\prime \prime}(v)(x, t) \\
\mathcal{B}_{\epsilon}^{\prime \prime}: L^{\infty}\left((0, T) ; \mathbb{K}_{d i v}^{1}\right) & \longrightarrow \mathcal{L}_{2}\left(\mathbb{X}_{v}^{0}, L^{2}\left((0, T) ; L^{2}\left(I ; \mathbb{R}^{3}\right)\right)\right) \\
v(x, t) & \longmapsto \mathcal{B}_{\epsilon}^{\prime \prime}(v)(x, t)
\end{aligned}
$$

Let the increases $h$ and $g$ be difined on $L^{2}\left((0, T) ; H^{1}\left(I ; \mathbb{R}^{3}\right)\right.$ ). We also note by $d\left[\mathcal{A}_{\epsilon}^{\prime}(v) g, h\right]$ and $d\left[\mathcal{B}_{\epsilon}^{\prime}(v) g, h\right]$ (for these notations seeTrenoguine, 1985), the second derivative of $\mathcal{A}_{\epsilon}(v)$ and $\mathcal{B}_{\epsilon}(v)$ in $v$ with $\mathcal{A}_{\epsilon}^{\prime}(v) g=d \mathcal{A}_{\epsilon}(v, g)$.

For an increase of $h$, independent of $g$, we have :

$$
\mathcal{A}_{\epsilon}^{\prime}(v+g) h-\mathcal{A}_{\epsilon}^{\prime}(v) h=\sum_{i=1}^{3} \partial_{w_{i}}^{2} \varphi \frac{\partial^{2} g h}{\partial x_{i} \partial t}+\partial_{v}^{2} \varphi g h+R_{\epsilon}(v, g h),
$$




$$
\mathcal{B}_{\epsilon}^{\prime}(v+g) h-\mathcal{B}_{\epsilon}^{\prime}(v) h=\sum_{i=1}^{3} \partial_{w_{i}}^{2} \vartheta \frac{\partial^{2} g h}{\partial x_{i} \partial t}+\partial_{v}^{2} \vartheta g h+R_{\epsilon}(v, g h) .
$$

For $h=g$ we deduce the following formulas

$$
\begin{aligned}
& d\left[\mathcal{A}_{\epsilon}^{\prime}(v) g, h\right]_{h=g}=\sum_{i=1}^{3} \partial_{w_{i}}^{2} \varphi \frac{\partial^{2} g^{2}}{\partial x_{i} \partial t}+\partial_{v}^{2} \varphi g^{2}, \\
& d\left[\mathcal{B}_{\epsilon}^{\prime}(v) g, h\right]_{h=g}=\sum_{i=1}^{3} \partial_{w_{i}}^{2} \vartheta \frac{\partial^{2} g^{2}}{\partial x_{i} \partial t}+\partial_{v}^{2} \vartheta g^{2} .
\end{aligned}
$$

Let us now give the definition of the generalized solution of the perturbed problem $(2.7)_{1}-(2.7)_{5}$.

Definition 1 Let $v_{0} \in \mathbb{K}_{d i v}^{1}, \rho_{0} \in W^{1,2}(I ; \mathbb{R})$. Generalized solution of the problem $(2.7)_{1}-(2.7)_{5}$ is a couple of functions $(v, \rho) \in L^{\infty}\left((0, T) ; \mathbb{K}_{d i v}^{1}\right) \times L^{\infty}\left((0, T) ; W^{1,2}(I ; \mathbb{R})\right)$ such as

1. integral equality is verified

$$
\begin{gathered}
\int_{0}^{T}\left\langle\rho \partial_{t} v, \mathcal{G}\right\rangle d t-\mu \int_{0}^{T}\langle\nabla v, \nabla \mathcal{G}\rangle d t-(\lambda+\mu) \int_{0}^{T}\langle\nabla v, \operatorname{div}(\mathcal{G})\rangle d t \\
+\int_{0}^{T} \int_{I}\left[\varphi_{v}(x, t, v, \nabla v)+\rho \int_{0}^{T} \int_{I} \mathcal{P}\left(x-y, t-t^{\prime}\right) \vartheta_{v}\left(y, t^{\prime}, v, \nabla v\right) d y d t\right] \mathcal{G} d x d t=\int_{0}^{T}\left\langle\rho \xi_{e}, \mathcal{G}\right\rangle d t
\end{gathered}
$$

for any $0 \leq t \leq T$ and $\mathcal{G} \in C\left((0, T) ; \mathbb{K}_{\text {div }}^{1}\right)$

2. the function $\rho(x, t)$ admits a generalized derivative following $(x, t)$. Moreover we have

$$
-\int_{0}^{T}\left\langle\rho, \frac{\partial \mathcal{y}}{\partial t}\right\rangle d t-\sum_{j=1}^{3} \int_{0}^{T}\left\langle\rho v_{j}, \frac{\partial \mathcal{y}}{\partial x_{j}}\right\rangle d t=\left\langle\rho_{0}(x), \mathcal{Y}(x, 0)\right\rangle
$$

for any $0 \leq t \leq T$ and $y \in C\left((0, T) ; W^{1,2}(I ; \mathbb{R})\right)$.

Theorem 2 (uniqueness of the solution of the perturbed system)

Let $v_{0} \in \mathbb{K}_{\text {div }}^{1}, \rho_{0} \in W^{1,2}(I ; \mathbb{R})$. There is a number $\zeta>0$ such that $0<\max \left(\beta^{-1}, \widetilde{\beta}^{-1}\right)<\zeta$ and suppose that the assumptions(H-1), (H-2) and (H-3) about functions $\varphi$ and $\vartheta$ are satisfied. Then the problem $(2.7)_{1}-(2.7)_{5}$ admits a unique solution $(\nu, \rho)=\mathfrak{R}_{\epsilon}\left(q_{0}, \xi_{e}\right)$ for every $\xi_{e} \in \mathbb{X}_{\xi_{e}}^{0}$. Morever, $\Re_{\epsilon}$ is $\mathscr{C}$-continuous and $\mathscr{C}$-differentiable. On the other hand, the operator $\Re_{\epsilon}$ is strongly differentiable on $L^{\frac{2 s}{s+1}}\left(I ; \mathbb{R}^{3}\right) \times \mathbb{X}_{\xi_{e}}^{0}$ as an application on space $\left(L^{2}\left((0, T) ; \mathbb{K}_{\text {div }}^{1}\right) ; \sigma\right)$ where $\sigma$ is the weak topology in $L^{2}\left((0, T) ; \mathbb{K}_{d i v}^{1}\right)$.

Let's introduce the space $\mathcal{V}(0, T)$

$\mathcal{V}(0, T):=\left\{v \in \mathbb{X}_{v}^{0}, \exists ! \xi_{e} \in \mathbb{X}_{\xi_{e}}^{0}, \exists ! q_{0} \in L^{\frac{2 s}{s+1}}\left(I ; \mathbb{R}^{3}\right), \frac{1}{\rho} L_{\lambda, \mu}(v)+\frac{\partial v}{\partial t} \equiv\left(q_{0}, \xi_{e}\right)\right\}$

Let's put $L_{\lambda, \mu}(v) \stackrel{\operatorname{def}}{=} \operatorname{div}(2 \mu \mathbf{D}[v]+\lambda \operatorname{div}(v)-\pi(\rho))$.

In (Gossan D, 2018), we had to show that when the functions $\varphi(x, t, v, w)$ and $\vartheta\left(y, t^{\prime}, v, w\right)$ satisfy the inequality (2.8) (2.11), the operators $\mathcal{A}_{\epsilon}^{\prime}(v)$ and $\mathcal{B}_{\epsilon}^{\prime}(v)$ are $\mathscr{C}$-continuous and $\mathscr{C}$-differentiable on $W(0, T)$ and satisfy the lipschitz condition, where

$$
W(0, T)=:\left\{\begin{array}{l|l}
\mathcal{A}_{\epsilon}(v) \in L^{2}\left((0, T) ; L^{2}\left(I ; \mathbb{R}^{3}\right)\right): \mid \begin{array}{l}
\mathcal{A}_{\epsilon}^{\prime}(v) \in \mathcal{L}\left(\mathbb{X}_{v}^{0}, L^{2}\left((0, T) ; L^{2}\left(I ; \mathbb{R}^{3}\right)\right)\right) \\
\mathcal{A}_{\epsilon}^{\prime \prime}(v) \in \mathcal{L}_{2}\left(\mathbb{X}_{v}^{0}, L^{2}\left((0, T) ; L^{2}\left(I ; \mathbb{R}^{3}\right)\right)\right)
\end{array}
\end{array}\right\} .
$$

Similarly, it has been prooved that, since $\mathcal{P}\left(x-y, t-t^{\prime}\right)$ is a continuous linear application and that operators $\mathcal{A}_{\epsilon}^{\prime}(v)$ and $\mathcal{B}_{\epsilon}^{\prime}(v)$ satisfy the Lipschitz condition, then using Hadamard's theorem, we can write that for all $v_{0} \in \mathcal{V}(0, T)$, the operator $\Re_{\epsilon}(V) \equiv\left(q_{0}, L_{\lambda, \mu}(v)+d\left[\mathcal{A}_{\epsilon}^{\prime}\left(v_{0}\right) h, g\right]_{h=g}+\int_{0}^{T} \int_{I} \mathcal{P} d\left[\mathcal{B}_{\epsilon}^{\prime}\left(v_{0}\right) g, h\right]_{h=g} d x d t\right)$ defined on $\mathcal{V}(0, T)$ with values in $\mathbb{X}_{\xi_{e}}^{0} \times L^{\frac{2 s}{s+1}}\left(I ; \mathbb{R}^{3}\right)$ admits a continuous inverse having the following form

$$
\mathfrak{R}_{\epsilon}^{-1}(v) \equiv\left(q_{0}, L_{\lambda, \mu}(v)+\mathcal{A}_{\epsilon}^{\prime}(v) g+\int_{0}^{T} \int_{I} \mathcal{P} \mathcal{B}^{\prime}{ }_{\epsilon}(v) g d x d t\right)
$$

of $\mathbb{X}_{\xi_{e}}^{0} \times L^{\frac{2 s}{s+1}}\left(I ; \mathbb{R}^{3}\right)$ in $\mathcal{V}(0, T)$ and besides, it is a homomorphism.

Remark 1 For some smooth conditions on the operator and using Hadamard's theorem on the strong differentiability of inverse functions, the operator $\mathfrak{R}_{\epsilon}(V)$ is strongly differentiable. This derivation is weaker than that of Fréchet. However, this allows us to establish the necessary conditions for optimal problems related to these equations. 


\section{Optimal Control Problem}

\subsection{The Formulation of the Optimal Control Problem}

The theory of optimal control of dynamic problems has important applications in both engineering and human science. Optimal control of a biological process in order to achieve a desired goal is important for many medical applications. In such optimal control problems, the control variable that makes the optimal state can be obtained by minimizing or maximizing a performance function. Moreover, the general problems of optimal control of non-convex costs are studied in depth for nonlinear systems by many researchers (Fattorini, 1996;Barbu, 1993; Li and Yong 1993 and the references cited therein). However, in practical applications to differential partial equations, there is some research involving initial value controls and the cost function attached is not necessarily non-convex. With this question in mind, we're studying the problems of optimal convex cost control for $(2.7)_{1}-(2.7)_{5}$. Let $\ell(v, \rho)$ and $\psi\left(\xi_{e}(x, t)\right)$ be to convex functions, respectively modelling the density of proliferating cells and necrotic cells (Jean Baptiste, 2003), at a time $t \leq T$. The cost $\mathcal{J}\left(v, \rho, \xi_{e}\right)$ attached to $(2.7)_{1}-(2.7)_{5}$ is given by the following general full cost.

$$
\begin{aligned}
\mathcal{J}: \mathbb{X}_{v}^{0} \times L^{\infty}\left((0, T) ; W^{1,2}\right) \times \mathbb{X}_{\xi_{e}}^{0} & \longrightarrow[0 ;+\infty] \\
\left(v, \rho, \xi_{e}\right) & \longmapsto \int_{0}^{T} \int_{I} \psi\left(\xi_{e}(x, t)\right) d x d t+\int_{0}^{T} \int_{I} \ell(v, \rho) d x d t,
\end{aligned}
$$

where $\psi: \mathbb{X}_{\xi_{e}}^{0} \longrightarrow \overline{\mathbb{R}}_{+}, \ell: \mathbb{X}_{v}^{0} \times L^{\infty}\left((0, T) ; W^{1,2}\right) \longrightarrow \overline{\mathbb{R}}_{+}$. We assume the following conditions on $\psi$ and $\ell$ in (3.1).

C1: The mapping $\xi_{e} \longmapsto \psi\left(\xi_{e}\right)$ is convex and semi continuous inferiorly.

C2: The mapping $\xi_{e} \longmapsto \psi\left(\xi_{e}\right)$ is locally lipschitzian,

$$
\forall T>0, \forall(x, t) \in I \times(0, T), \forall \xi_{e}, \hat{\xi}_{e} \in \mathbb{X}_{\xi_{e}}^{0}
$$

$$
\exists \eta_{1}>0:\left|\psi\left(\hat{\xi}_{e}\right)-\psi\left(\xi_{e}\right)\right| \leq \eta_{1}\left|\hat{\xi}_{e}-\xi_{e}\right| .
$$

C3: The mapping $(v, \rho) \longmapsto \ell(v, \rho)$ is convex and semi continuous inferiorly.

C4: The mapping $(v, \rho) \longmapsto \ell(v, \rho)$ is locally lipschitzian,

$$
\forall T>0, \forall(x, t) \in I \times(0, T), \forall(v, \rho) ;(\hat{v}, \hat{\rho}) \in \mathbb{X}_{v}^{0} \times L^{\infty}\left((0, T) ; W^{1,2}\right)
$$

$$
\exists \eta_{2}>0:|\ell(\hat{v}, \hat{\rho})-\ell(v, \rho)| \leq \eta_{2}(|\hat{v}-v|+|\hat{\rho}-\rho|) .
$$

The main objective is to establish the existence of optimal control that minimizes the functional cost (3.1) subject to the $(2.7)_{1}-(2.7)_{5}$ constraint and to prove the necessary first-order optimality condition using the variational principle and the Fréchet differentiability of the functional.

Definition 3 Let $\hat{X}^{a d}$ be a closed and convex subset composed of controls $\xi_{e} \in L^{1}\left((0, T) ; L^{\frac{2 s}{s-1}}\left(I ; \mathbb{R}^{3}\right)\right)$.

Definition 4 The permissible $\hat{\mathcal{U}}^{\text {ad }}$ class of the triplet $\left(v, \rho, \xi_{e}\right)$ is defined as the set of states $(v, \rho)$ with the initial data $v_{0} \in \mathbb{K}_{\text {div }}^{1}$ and $\rho_{0} \in W^{1,2}(I ; \mathbb{R})$, resolving the system $(2.7)_{1}-(2.7)_{5}$ with control $\xi_{e} \in \hat{X}^{a d}$. Which is to say

$$
\hat{\mathcal{U}}^{\text {ad }}: \stackrel{\text { def }}{=}\left\{\left(v, \rho, \xi_{e}\right):(v, \rho) \text { is the unique solution of }(2.7)_{1}-(2.7)_{5} \text { with control } \xi_{e}\right\} .
$$

The optimal control problem that we will study in this paper is as follows

$$
\mathcal{J}\left(v^{*}, \rho^{*}, \xi_{e}^{*}\right)=\min _{\left(v, \rho, \xi_{e}\right) \in \hat{\mathcal{U}}^{a d}} \mathcal{J}\left(v, \rho, \xi_{e}\right),
$$

and that constraints given in the form of equality and inequality are verified

$$
\begin{cases}\mathcal{J}^{(k)}\left(\xi_{e}\right) \leq 0, & k=0, \cdots, p \\ \mathcal{J}^{(k)}\left(\xi_{e}\right)=0, & k=p+1, \cdots, q\end{cases}
$$

Definition 5 A solution to the problem (3.2), called an optimal solution and the optimal triplet, is denoted by $\left(v^{*}, \rho^{*}, \xi_{e}^{*}\right)$. The control $\xi_{e}^{*}$ is called an optimal control, i.e. a control corresponding to the best cost.

\subsection{Existence and Uniqueness of Optimal Control}

In this section, we use the notion of minimizing sequences to prove the existence and uniqueness of an optimal control $\left(v^{*}, \rho^{*}, \xi_{e}^{*}\right)$ for the functional (3.1) in $\hat{\mathcal{U}}^{a d}$. This is the content of the following theorem. 
Theorem 6 Suppose the assumptions of thetheorem 2 and thetheorem 14 of (Gossan, 2018) are verified. Then there is only one optimal solution $\left(v^{*}, \rho^{*}, \xi_{e}^{*}\right) \in \hat{\mathcal{U}}^{\text {ad }}$ such that the functional $\mathcal{J}\left(v, \rho, \xi_{e}\right)$ reaches its minimum at $\left(v^{*}, \rho^{*}, \xi_{e}^{*}\right)$ where $\left(v^{*}, \rho^{*}\right)$ is the unique general solution for the control system $(2.7)_{1}-(2.7)_{5}$ with control $\xi_{e}^{*}$.

Proof. Step $1:\left(\hat{\mathcal{U}}^{a d} \neq \varnothing\right)$.

Indeed, $\mathcal{J}$ is a closed convex set, there is a minimizing sequence $\left(v_{n}, \rho_{n}, \xi_{\text {en }}\right)$ in $\hat{\mathcal{U}}^{\text {ad }}$ such that

$$
\inf _{\xi_{e} \in \hat{\mathcal{U}}^{a d}} \mathcal{J}\left(v, \rho, \xi_{e}\right)=\lim _{n \rightarrow+\infty} \mathcal{J}\left(v_{n}, \rho_{n}, \xi_{\text {en }}\right) .
$$

So $\mathcal{J}\left(\left(v_{n}, \rho_{n}, \xi_{\text {en }}\right)\right)<+\infty$. Therefore $\hat{\mathcal{U}}^{\text {ad }}$ is not empty.

Step 2 : Let's show that any minimal sequence of controls is bounded. Let put

$$
\overline{\mathcal{J}}=\inf _{\xi_{e} \in \hat{\mathcal{U}}^{a d}} \mathcal{J}\left(v, \rho, \xi_{e}\right)
$$

As $0 \leq \overline{\mathcal{J}}<+\infty$, there is a minimizing sequence $\left\{\xi_{\text {en }}\right\}_{n \in \mathbb{N}} \in \hat{\mathcal{U}}^{\text {ad }}$ such as

$$
\lim _{n \rightarrow+\infty} \mathcal{J}\left(v_{n}, \rho_{n}, \xi_{\text {en }}\right)=\overline{\mathcal{J}}
$$

But $\left(v_{n}, \rho_{n}\right)$ is the unique solution of $(2.7)_{1}-(2.7)_{5}$ with control $\xi_{\text {en }}$ and $v_{n}(0)=v_{0 n} \in \mathbb{K}_{\text {div }}^{1}$ and $\rho_{n}(0)=\rho_{0 n} \in W^{1,2}(I ; \mathbb{R})$. Suppose that $\mathcal{J}\left(v_{n}, \rho_{n}, \xi_{\text {en }}\right) \leq \mathcal{J}(v, \rho, 0)$, where $(v, \rho, 0) \in \hat{\mathcal{U}}^{a d}$. From the definition of $\mathcal{J}(., .,$.$) it follows that$

$$
\int_{0}^{T} \int_{I} \psi\left(\xi_{\text {en }}\right) d x d t+\int_{0}^{T} \int_{I} \ell\left(v_{n}, \rho_{n}\right) d x d t \leq \int_{0}^{T} \int_{I} \ell(v, \rho) d x d t .
$$

From this report it is evident that there is a constant $d_{0}>0$ big enough such that

$$
\int_{0}^{T} \int_{I} \psi\left(\xi_{e n}\right) d x d t \leq \mathcal{J}\left(v_{n}, \rho_{n}, \xi_{e n}\right) \leq d_{0} .
$$

Therefore the sequence $\xi_{\text {en }}$ is uniformly bounded in the space $L^{1}\left((0, T) ; L^{\frac{2 s}{s-1}}\left(I ; \mathbb{R}^{3}\right)\right)$. Since $\left(v_{n}, \rho_{n}\right)$ is a general solution of the system $(2.7)_{1}-(2.7)_{5}$ with control $\xi_{\text {en }}$, we can easily show that $v_{n}$ is uniformly bounded in $\operatorname{space} L^{\infty}\left((0, T) ; \mathbb{K}_{d i v}^{1}\right) \cap$ $L^{2}\left((0, T) ; \mathbb{K}_{d i v}^{0}\right)$ and $\rho_{n}$ is uniformly bounded in space $L^{\infty}\left((0, T) ; W^{1,2}(I ; \mathbb{R})\right)$.

So using Banach-Alaoglu theorem (Brezis, 1999) we can extract a subsequence $\left\{\left(v_{n_{k}}, \rho_{n_{k}}, \xi_{\text {en }}\right)\right\} \subset\left\{\left(v_{n}, \rho_{n}, \xi_{\text {en }}\right)\right\}$ such that

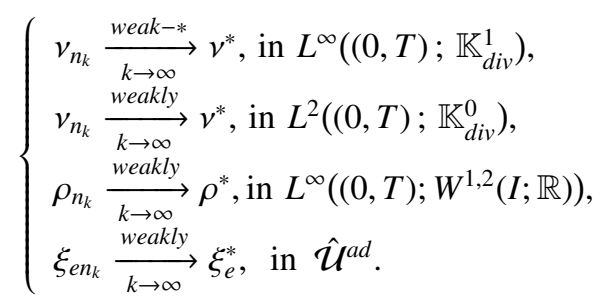

Using the compacity-results introduced by Aubin Lions, we can get from (3.5) that $v_{n_{k}} \in L^{\infty}\left((0, T) ; \mathbb{K}_{d i v}^{0}\right) \cap L^{2}\left((0, T) ; \mathbb{K}_{d i v}^{1}\right)$ and $\rho_{n_{k}} \in L^{2}\left((0, T) ; W^{1,2}(I ; \mathbb{R})\right)$. Thus again, using Banach- Alaoglu theorem we can deduce that

$$
\left\{\begin{array}{l}
v^{*} \in C\left((0, T) ; \mathbb{K}_{d i v}^{0}\right), \\
\rho^{*} \in C\left((0, T) ; W^{1,2}(I ; \mathbb{R})\right), \\
v_{0}^{*} \in \mathbb{K}_{d i v}^{0}, \\
\rho_{0}^{*} \in W^{1,2}(I ; \mathbb{R}) .
\end{array}\right.
$$

Therefore $\left(v^{*}, \rho^{*}, \xi_{e}^{*}\right)$ is the unique solution of $(2.7)_{1}-(2.7)_{5}$ with control $\xi_{e}^{*} \in \hat{\mathcal{U}}^{a d}$. It implies that $\left(v^{*}, \rho^{*}, \xi_{e}^{*}\right) \in \hat{\mathcal{U}}^{a d}$.

Step 3. It remains to show that the limit $\left(v^{*}, \rho^{*}, \xi_{e}^{*}\right)$ is an optimal triplet. By theassumptions $\mathbf{C 1}$ and $\mathbf{C 3}$, the functions $\psi$ and $\ell$ are lower semi-continuous functions and convex so they are weakly continuous inferiorly. Since the cost function $\mathcal{J}$ is convex on $L^{2}\left((0, T) ; \mathbb{K}_{\text {div }}^{0}\right) \times L^{2}\left((0, T) ; W^{1,2}(I ; \mathbb{R})\right) \times \hat{X}^{a d}$, let's show that $\mathcal{J}$ is lower semi-continuous. Let $\left(v_{n}, \rho_{n}, \xi_{\text {en }}\right)$ be a sequence converging weakly to $\left(\nu, \rho, \xi_{e}\right)$ in $L^{2}\left((0, T) ; \mathbb{K}_{\text {div }}^{0}\right) \times L^{2}\left((0, T) ; W^{1,2}(I ; \mathbb{R})\right) \times L^{1}\left((0, T) ; L^{\frac{2 s}{s-1}}\left(I ; \mathbb{R}^{3}\right)\right)$. The lower semi-continuity of $\psi$ and $\ell$ results in

$$
\psi\left(\xi_{e}\right)+\ell(v, \rho) \leq \lim _{n \rightarrow \infty} \inf \left[\psi\left(\xi_{e n}\right)+\ell\left(v_{n}, \rho_{n}\right)\right] .
$$


The function $\lim _{n \rightarrow \infty} \inf \left[\psi\left(\xi_{e n}\right)+\ell\left(v_{n}, \rho_{n}\right)\right] \in L^{1}\left(I_{t}\right)$ is integrable and

$$
\int_{I_{t}} \lim _{n \rightarrow \infty} \inf \left[\psi\left(\xi_{e n}\right)+\ell\left(v_{n}, \rho_{n}\right)\right] \leq \lim _{n \rightarrow \infty} \inf \int_{I_{t}}\left[\psi\left(\xi_{e n}\right)+\ell\left(v_{n}, \rho_{n}\right)\right]
$$

The hypotheses of lower semi-continuity make it possible to conclude that $\mathcal{J}$ is lower semi-continuous. Returning to the minimizing sequence we deduce that

$$
\lim _{n \rightarrow \infty} \inf \mathcal{J}\left(v_{n}, \rho_{n}, \xi_{e n}\right) \geqslant \mathcal{J}\left(v^{*}, \rho^{*}, \xi_{e}^{*}\right) .
$$

As from (3.9),we obtain

$$
\mathcal{J}\left(v^{*}, \rho^{*}, \xi_{e}^{*}\right) \leq \lim _{n \rightarrow \infty} \inf \mathcal{J}\left(v_{n}, \rho_{n}, \xi_{e n}\right)=\inf _{\left(v, \rho, \xi_{e}\right) \in \hat{\mathcal{U}}^{a d}} \mathcal{J}\left(v, \rho, \xi_{e}\right) .
$$

Moreover, taking in to account the fact that $\inf _{\left(\nu, \rho, \xi_{e}\right) \in \hat{\mathcal{U}}^{a d}} \mathcal{J}\left(v, \rho, \xi_{e}\right) \leq \mathcal{J}\left(v^{*}, \rho^{*}, \xi_{e}^{*}\right)$ by definition, we can deduce that

$$
\mathcal{J}\left(v^{*}, \rho^{*}, \xi_{e}^{*}\right)=\mathcal{J}\left(\xi_{e}^{*}\right)=\inf f_{\left(v, \rho, \xi_{e}\right)}=\overline{\mathcal{J}} .
$$

Uniquess: Since $\mathcal{J}$ is strictly convex, the minimum is unique. Thus, the problem admits a unique solution. Indeed, let $\xi_{e}^{*} \in \hat{\mathcal{U}}^{a d}$ and $\widetilde{\xi}_{e}^{*} \in \hat{\mathcal{U}}^{a d}$ be two optimal controls, which respectively satisfy

$$
\begin{aligned}
& \mathcal{J}\left(\xi_{e}^{*}\right)=\min _{\xi_{e} \in \hat{\mathcal{U}}^{a d}} \mathcal{J}\left(\xi_{e}\right), \\
& \mathcal{J}\left(\widetilde{\xi}_{e}^{*}\right)=\min _{\xi_{e} \in \hat{\mathcal{U}}^{a d}} \mathcal{J}\left(\xi_{e}\right) .
\end{aligned}
$$

As $\hat{\mathcal{U}}^{a d}$ is a convex and not empty admissible set, then for $\epsilon \in(0 ; 1)$ we can get the following

$$
\epsilon \xi_{e}^{*}+(1-\epsilon) \widetilde{\xi}_{e}^{*} \in \hat{\mathcal{U}}^{a d}
$$

We therefore deduce that

which is a contradiction unless

$$
\begin{aligned}
& \mathcal{J}\left[\eta \xi_{e}^{*}+(1-\epsilon) \widetilde{\xi}_{e}^{*}\right]<\epsilon \mathcal{J}\left(\xi_{e}^{*}\right)+(1-\epsilon) \mathcal{J} \widetilde{\xi}_{e}^{*}, \\
= & \min _{\xi_{e} \in \hat{\mathcal{U}}^{a d}} \mathcal{J}\left(\xi_{e}\right),
\end{aligned}
$$

$$
\xi_{e}^{*}=\widetilde{\xi}_{e}^{*}
$$

This completes the proof.

Theorem 7 (Stability of the solution in the context of a control perturbation)

Let's $\xi_{e}, \xi_{e}^{\varepsilon} \in L^{2}\left((0, T) ; L^{\frac{2 s}{s-1}}\left(I ; \mathbb{R}^{3}\right)\right)$. If the pair of functions $(v, \rho)\left(\right.$ resp. $\left.\left(v_{\varepsilon}, \rho_{\varepsilon}\right)\right)$ is a solution of the system $(2.7)_{1}-(2.7)_{5}$ corresponding to the command $\xi_{e}\left(\right.$ resp. $\left.\left(v_{\varepsilon}\right)\right)$, then for $\delta v=v_{\varepsilon}-v, \delta \rho=\rho_{\varepsilon}-\rho$ and $\delta \xi_{e}=\xi_{e}^{\varepsilon}-\xi_{e}$, we have the following estimates

$$
\begin{aligned}
& \|\delta v\|_{L^{2}\left(I ; \mathbb{R}^{3}\right)} \leq d_{1}\left\|\delta \xi_{e}\right\|_{\mathbb{X}_{\mathbb{\xi}_{e}}^{0}} \\
& \|\delta \rho\|_{W^{1,2(I ; \mathbb{R})}} \leq d_{2}\left\|\delta \xi_{e}\right\|_{\mathbb{X}_{\mathbb{\xi}_{e}}^{0}}
\end{aligned}
$$

where $d_{i}(i=1.2)$ independent of $\delta \xi_{e}$.

Proof. The estimation (3.11) is obtained from the fact that $\delta v$ and $\delta \xi_{e}$ satisfy the estimate

$$
\|v\|_{L^{2}\left(I ; \mathbb{R}^{3}\right)}^{2} \leq \beta^{-1}\left[\left(\left\|q_{0}\right\|_{L^{\frac{2 s}{s+1}\left(I ; \mathbb{R}^{3}\right)}}^{2}+\left\|\xi_{e}\right\|_{\mathbb{X}_{\xi_{e}}^{0}}^{2}\right) \exp (T)\right] .
$$

Thereafter at $t=0$ for $q_{0}=0$, we get the result. The estimation (3.12) is obtained using the mark-up (3.19) of lemma 4 (see Gossan D, 2018) and from inequality (3.11) we obtain the estimate (3.12). 


\section{Formulation of the Functional Gradient}

\subsection{Adjoint System}

As it is well known from the literature of control theory, in order to obtain the necessary conditions of optimality, we need the adjoint equations corresponding to the system $(2.7)_{1}-(2.7)_{5}$. Using thetheorem 6 we obtain the following result.

Theorem 8 There is an optimal control $\xi_{e}^{*}$ and a corresponding solution $\left(v^{*}, \rho^{*}\right)$ such that

$$
\mathcal{J}\left(v^{*}, \rho^{*}, \xi_{e}^{*}\right)=\min _{\left(v, \rho, \xi_{e}\right) \in \hat{\mathcal{U}}^{a d}} \mathcal{J}\left(v, \rho, \xi_{e}\right)
$$

with $\mathcal{J}\left(\xi_{e}\right):=\int_{0}^{T} \int_{I} \psi\left(\xi_{e}(x, t)\right) d x d t+\int_{0}^{T} \int_{I} \ell(v(x, t), \rho(x, t)) d x d t$.

Moreover, it exists adjoint variables $(\mathcal{G}, \mathcal{Y}) \in C\left((0, T) ; \mathbb{K}_{\text {div }}^{1}\right) \times C\left((0, T) ; W^{1,2}(I ; \mathbb{R})\right)$ solution of the following dual problem

$$
\left\{\begin{array}{cl}
-\partial_{t} \mathcal{G}-\rho^{-1}(2 \mu+\lambda) \Delta \mathcal{G}+\left(\mathcal{A}_{\epsilon}^{\prime \prime}(v)+\mathcal{P}^{*} \mathcal{B}_{\epsilon}^{\prime \prime}(v)\right) \mathcal{G}-\nabla^{T} \rho \mathcal{Y} & =-\ell_{v}, \\
-\partial_{t} \mathcal{Y}-\nu \nabla \mathcal{Y} & =-\ell_{\rho}, \\
\xi_{e}(x, t) & =\mathcal{G}(x, t), \\
\sum_{j=1}^{3} \frac{\partial \mathcal{G}_{j}}{\partial x_{j}} & =0 \\
\left.\mathcal{G}\right|_{\partial I}=\left.\mathcal{Y}\right|_{\partial I} & =0 \\
\mathcal{G}(., T)=\mathcal{Y}(., T) & =0 .
\end{array}\right.
$$

Proof. Let's introduce a new functional $L\left(v, \rho, \xi_{e}, \mathcal{G}, \mathcal{Y}\right)$, associated with the functional cost $\mathcal{J}$ defined in (3.1) by

$$
L\left(v, \rho, \xi_{e}, \mathcal{G}, \mathcal{Y}\right):=\mathcal{J}\left(v, \rho, \xi_{e}\right)+\int_{0}^{T} \int_{I}\left(\partial_{t} v-\mathcal{M}_{1}\right) \mathcal{G} d x d t+\int_{0}^{T} \int_{I}\left(\partial_{t} \rho-\mathcal{M}_{2}\right) \mathcal{y} d x d t
$$

where $\mathcal{G}$ and $\mathcal{Y}$ denote the variables associated with $v$ and $\rho$ respectively. The functions $\mathcal{M}_{1}$ and $\mathcal{M}_{2}$ are defined as follows,

$$
\left\{\begin{array}{l}
\mathcal{M}_{1} \stackrel{\text { def }}{:}-\widetilde{F}(\mathcal{H}, \varphi)-\rho^{-1} \nabla \pi+\xi_{e}+\rho^{-1}\left[\mu \operatorname{div}(\nabla v)+(\lambda+\mu) \nabla^{2} v\right], \\
\mathcal{M}_{2} \stackrel{\text { def }}{=}-\sum_{j=1}^{3} \rho \frac{\partial v_{j}}{\partial x_{j}} .
\end{array}\right.
$$

We derive the functional (4.2) in the sense of Fréchet with respect to variables $\left(v, \rho, \xi_{e}\right)$ and we get the following system.

$$
\left\{\begin{array}{l}
\frac{\partial \mathcal{J}\left(v, \rho, \xi_{e}\right)}{\partial v}+\int_{0}^{T} \int_{I}\left(\partial_{t} v-\partial_{v} \mathcal{M}_{1}\right) \mathcal{G} d x d t+\int_{0}^{T} \int_{I}\left(\partial_{t} \rho-\partial_{v} \mathcal{M}_{2}\right) \mathcal{y} d x d t=0 \\
\frac{\partial \mathcal{J}\left(v, \rho, \xi_{e}\right)}{\partial \rho}+\int_{0}^{T} \int_{I}\left(\partial_{t} v-\partial_{\rho} \mathcal{M}_{1}\right) \mathcal{G} d x d t+\int_{0}^{T} \int_{I}\left(\partial_{t} \rho-\partial_{\rho} \mathcal{M}_{2}\right) \mathcal{y} d x d t=0 \\
\frac{\partial \mathcal{J}\left(v, \rho, \xi_{e}\right)}{\partial \xi_{e}}+\int_{0}^{T} \int_{I}\left(\partial_{t} v-\partial_{\xi_{e}} \mathcal{M}_{1}\right) \mathcal{G} d x d t+\int_{0}^{T} \int_{I}\left(\partial_{t} \rho-\partial_{\xi_{e}} \mathcal{M}_{2}\right) \mathcal{y} d x d t=0
\end{array}\right.
$$

Next, the adjoint variables $\mathcal{G}, \mathcal{Y}$ and $\xi_{e}$ satisfy the following system

$$
\left\{\begin{aligned}
-\partial_{t} \mathcal{G}-\rho^{-1}(2 \mu+\lambda) \Delta \mathcal{G}+\left(\mathcal{A}_{\epsilon}^{\prime \prime}(v)+\mathcal{P}^{*} \mathcal{B}_{\epsilon}^{\prime \prime}(v)\right) \mathcal{G}-(\nabla \rho)^{T} \mathcal{Y} & =-\frac{\partial \mathcal{J}}{\partial v}, \\
-\partial_{t} \mathcal{Y}+\rho^{-2}(2 \mu+\lambda) \nabla v \nabla \mathcal{G}-v \nabla \mathcal{Y} & =-\frac{\partial \mathcal{J}}{\partial \rho}, \\
\xi_{e}(x, y) & =\mathcal{G}(x, y), \\
\sum_{j=1}^{3} \frac{\partial \mathcal{G}_{j}}{\partial x_{j}} & =0, \\
\left.\mathcal{G}\right|_{\partial I}=\left.\mathcal{Y}\right|_{\partial I} & =0, \\
\mathcal{G}(., T)=\mathcal{Y}(., T) & =0 .
\end{aligned}\right.
$$


Thus, from (4.5) and taking into account the fact that $\nabla \mathcal{G}=0$, it follows that the adjoint variables $(\mathcal{G}, \mathcal{Y})$ satisfy the following adjoint system

$$
\left\{\begin{array}{cc}
-\partial_{t} \mathcal{G}-\rho^{-1}(2 \mu+\lambda) \Delta \mathcal{G}+\left(\mathcal{A}_{\epsilon}^{\prime \prime}(v)+\mathcal{P}^{*} \mathcal{B}_{\epsilon}^{\prime \prime}(v)\right) \mathcal{G}-\nabla^{T} \rho \mathcal{Y} & =-\ell_{\nu}, \\
-\partial_{t} \mathcal{Y}-v \nabla \mathcal{Y} & =-\ell_{\rho}, \\
\xi_{e}(x, t) & =\mathcal{G}(x, t), \\
\sum_{j=1}^{3} \frac{\partial \mathcal{G}_{j}}{\partial x_{j}} & =0, \\
\left.\mathcal{G}\right|_{\partial I}=\left.\mathcal{Y}\right|_{\partial I} & =0, \\
\mathcal{G}(., T)=\mathcal{Y}(., T) & =0 .
\end{array}\right.
$$

\subsection{Variation Calculation}

The calculation of variations studies the optimal form, time, speed, energy, volume etc.. The laws of physics of astronomical mechanics, as well as all natural and technical sciences obey variational principles. The main purpose of calculating the variations is to find the solution governed by these principles. The calculation of variations has a long history, and is renewed according to developments in mathematics and other sciences. These calculations make it possible to establish the necessary optimality conditions to solve this type of problem. For this, let's state the theorem following the Fréchet differentiability of the functional and the dual system of the problem $(2.7)_{1}-(2.7)_{5}$.

Theorem 9 Suppose all the conditions of the Definition 6 are verified. Let $\mathcal{A}_{\epsilon}$ and $\mathcal{B}_{\epsilon}$ be two differentiable nonlinear operators and $\psi$, differentiable following $\xi_{e}$ and its derivative $\psi_{\xi_{e}}$ checks the Lipchitz conditions following $\xi_{e}$. The function $\ell$ is differentiable wich respect to $v$ and $\rho$, and the partial derivative $\ell_{\rho}$ and $\ell_{v}$, fulfill the Lipchitz condition from $v$ and $\rho$. Then the functional $\mathcal{J}\left(\xi_{e}\right)$ is differentiable and its gradient is determined by the formula:

$$
\mathcal{J}^{\prime}\left(\xi_{e}\right)=\int_{0}^{T}\left(\psi_{\xi_{e}}\left(\xi_{e}\right)+\mathcal{G}\right) d t, \quad \forall x \in I
$$

Proof. Suppose that all the hypotheses of theorem 9 on functions $\psi$ and $\ell(v, \rho)$ are verified. Then let's examine the problem $(2.7)_{1}-(2.7)_{5}$ with perturbed command $\xi_{e}^{\varepsilon}(x, t)$ that would be linked to the solution $\left(v_{\varepsilon}, \rho_{\varepsilon}\right)$ of this problem and the value of the functional $\mathcal{J}\left(\xi_{e}^{\varepsilon}\right)$. For that let us consider the following increases:

$$
\left\{\begin{array}{l}
\delta \xi_{e}=\xi_{e}^{\varepsilon}-\xi_{e}+O(\varepsilon), \\
\delta v=v_{\varepsilon}-v+O(\varepsilon) \\
\delta \rho=\rho_{\varepsilon}-\rho+O(\varepsilon)
\end{array}\right.
$$

The increase in functional $\mathcal{J}\left(\xi_{e}^{\varepsilon}\right)$ is written :

$$
\begin{aligned}
\Delta \mathcal{J}\left(\xi_{e}\right) & =\mathcal{J}\left(\xi_{e}+\delta \xi_{e}\right)-\mathcal{J}\left(\xi_{e}\right) \\
& =\int_{0}^{T} \int_{I} \psi\left(\xi_{e}^{\varepsilon}\right) d x+\int_{0}^{T} \int_{I} \ell\left(v_{\epsilon}, \rho_{\varepsilon}\right) d x d t-\int_{0}^{T} \int_{I} \ell(v, \rho) d x d t-\int_{0}^{T} \int_{I} \psi\left(\xi_{e}\right) d x+O(\varepsilon) \\
& =\int_{0}^{T} \int_{I}\left[\psi\left(\xi_{e}^{\varepsilon}\right)-\psi\left(\xi_{e}\right)\right] d x+\int_{0}^{T} \int_{I}\left[\ell\left(v_{\varepsilon}, \rho_{\varepsilon}\right)-\ell(v, \rho)\right] d x d t+O(\varepsilon) \\
& =\int_{0}^{T} \int_{I} \psi_{\xi_{e}}\left(\xi_{e}\right) \delta \xi_{e} d x+\int_{0}^{T} \int_{I}\left[\ell_{v}(v, \rho) \delta v+\ell_{\rho}(v, \rho) \delta \rho\right] d x d t \\
& +\int_{0}^{T} \int_{I} \int_{0}^{1}\left[\left(\ell_{v}(v+\tau \delta v, \rho+\tau \delta \rho)-\ell_{v}(v, \rho)\right) \delta v\right] \\
& +\int_{0}^{T} \int_{I} \int_{0}^{1}\left[\left(\ell_{\rho}(v+\tau \delta v, \rho+\tau \delta \rho)-\ell_{\rho}(v, \rho)\right) \delta \rho\right] d \tau d x d t \\
& +\int_{0}^{T} \int_{I} \int_{0}^{1}\left(\psi_{\xi_{e}}\left(\xi_{e}+\tau \delta \xi_{e}\right)-\psi_{\xi_{e}}\left(\xi_{e}\right)\right) \delta \xi_{e} d \tau d x+O(\varepsilon)
\end{aligned}
$$


Let's put :

$$
\begin{gathered}
\mathcal{I}_{1}=\int_{0}^{1}\left[\left(\ell_{v}(v+\tau \delta v, \rho+\tau \delta \rho)-\ell_{v}(v, \rho)\right) \delta v\right] d \tau \\
\mathcal{I}_{2}=\int_{0}^{1}\left[\left(\ell_{\rho}(v+\tau \delta v, \rho+\tau \delta \rho)-\ell_{\rho}(v, \rho)\right) \delta \rho\right] d \tau \\
\mathcal{I}_{3}=\int_{0}^{1}\left(\psi_{\xi_{e}}\left(\xi_{e}+\tau \delta \xi_{e}\right)-\psi_{\xi_{e}}\left(\xi_{e}\right)\right) \delta \xi_{e} d \tau d x \\
\Delta \mathcal{J}\left(\xi_{e}\right)=\int_{0}^{T} \int_{I}\left[\ell_{v}(v, \rho) \delta v+\ell_{\rho}(v, \rho) \delta \rho\right] d x d t+\int_{0}^{T} \int_{I} \mathcal{I}_{1} d x d t \\
+\int_{0}^{T} \int_{I} \psi_{\xi_{e}}\left(\xi_{e}\right) \delta \xi_{e} d x d t+\int_{0}^{T} \int_{I} I_{2} d x d t+\int_{0}^{T} \int_{I} I_{3} d x d t .
\end{gathered}
$$

Taking into account (2.16)-(2.17) and the fact that the small variations $\delta v, \delta \rho$ and $\delta \xi_{\text {e }}$ verify these integrable equalities, the functional increase becomes :

$$
\begin{aligned}
\Delta \mathcal{J}\left[\xi_{e}\right] & =\int_{0}^{T} \int_{I}\left[\ell_{v}(v, \rho) \delta v+\ell_{\rho}(v, \rho) \delta \rho\right] d x d t+\int_{0}^{T}\left(\int_{I} \mathcal{I}_{1} d x+\int_{I} \mathcal{I}_{2} d x+\int_{I} \mathcal{I}_{3} d x\right) d t \\
& +\int_{0}^{T}\left\langle-\partial_{t} \mathcal{G}+\rho^{-1}(\lambda+2 \mu) \Delta \mathcal{G}+\left(\delta \varphi_{v}+\int_{0}^{T} \int_{I} \mathcal{P} \vartheta_{v} d y d t\right) \mathcal{G}+\nabla^{T} \rho \mathcal{G}, \delta v\right\rangle d t \\
& +\int_{0}^{T} \int_{I} \psi_{\xi_{e}}\left(\xi_{e}\right) \delta \xi_{e} d x-\int_{0}^{T}\left\langle\mathcal{G}, \delta \xi_{e}\right\rangle d t+\int_{0}^{T}\left\langle-\partial_{t} \mathcal{Y}-\nu \nabla \mathcal{Y}, \delta \rho\right\rangle d t
\end{aligned}
$$

Using the formulas(2.14) and (2.15), we obtain the following

$$
\begin{aligned}
& \int_{0}^{T} \int_{I} \delta \varphi_{\nu} \mathcal{G} d x d t=\int_{0}^{T} \int_{I}\left(\varphi_{\nu}\left(v_{\varepsilon}, \nabla v_{\varepsilon}\right)-\varphi_{v}(v, \nabla v)\right) \mathcal{G} d x d t \\
& =\int_{0}^{T} \int_{I} \varphi_{v v}(v) \mathcal{G} \delta v d x d t+\int_{0}^{T} \int_{I}\left(\int_{0}^{1}\left[\varphi_{v v}(v+\tau \delta v)-\varphi_{v v}(v)\right] \delta v d \tau\right) \mathcal{G} d x d t \\
& =\int_{0}^{T} \int_{I} d\left[\mathcal{A}_{\varepsilon}^{\prime}(v) h, g\right]_{g=h} \delta v \mathcal{G} d x d t \\
& +\underbrace{\int_{0}^{T} \int_{I}\left(\int_{0}^{1}\left[\varphi_{v v}(v+\tau \delta v)-\varphi_{v v}(v)\right] \delta v d \tau\right) \mathcal{G} d x d t}_{I_{4}} . \\
& \int_{0}^{T} \int_{I}\left(\int_{0}^{T} \int_{I} \mathcal{P} \delta \vartheta_{v} d y d t\right) \mathcal{G} d x d t=\int_{0}^{T} \int_{I}\left[\int_{0}^{T} \int_{I}\left(\int_{0}^{1} \vartheta_{v v}(v+\tau \delta v) \delta v(y) d \tau\right) \mathcal{P} d y d t\right] \mathcal{G} d x d t \\
& =\int_{I}\left[\int_{0}^{T} \int_{I} \vartheta_{v v}(v) \delta v(y) \mathcal{P} d y d t\right] \mathcal{G} d x \\
& +\underbrace{\int_{0}^{T} \int_{I}\left[\int_{0}^{T} \int_{I} \int_{0}^{1}\left(\vartheta_{v v}(v+\tau \delta v)-\vartheta_{v v}(v)\right) \delta v(y) \mathcal{P} d \tau d y d t\right] \mathcal{G} d x d t}_{I_{5}} \\
& =\int_{0}^{T} \int_{I} d\left[\mathcal{B}_{\varepsilon}^{\prime}(v) h, g\right]_{h=g} \mathcal{P}^{*} \delta v \mathcal{G}^{T} d x d t+\mathcal{I}_{4}
\end{aligned}
$$

Due to the results(4.11) and (4.12), we deduce that 


$$
\begin{aligned}
\Delta \mathcal{J}\left(\xi_{e}\right) & =\int_{0}^{T} \int_{I}\left[\ell_{v}(v, \rho) \delta v+\ell_{\rho}(v, \rho) \delta \rho\right] d x d t+\int_{0}^{T} \int_{I} \psi_{\xi_{e}}\left(\xi_{e}\right) \delta \xi_{e} d x d t \\
& +\int_{0}^{T}\left(\int_{I} \mathcal{I}_{1} d x+\int_{I} \mathcal{I}_{2} d x+\int_{I} \mathcal{I}_{2} d x\right) d t-\int_{0}^{T}\left\langle\mathcal{G}, \delta \xi_{e}\right\rangle d t+\mathcal{I}_{4}+\mathcal{I}_{5} \\
& +\int_{0}^{T}\left\langle\rho^{-1}(\lambda+2 \mu) \Delta \mathcal{G}+d\left[\mathcal{A}_{\varepsilon}^{\prime}(v) h, g\right]_{g=h} \mathcal{G}+d\left[\mathcal{B}_{\varepsilon}^{\prime}(v) g, h\right]_{g=h} \mathcal{P}^{*} \mathcal{G}, \delta v\right\rangle d t \\
& =\int_{0}^{T} \int_{I}\left[\ell_{v}(v, \rho) \delta v+\ell_{\rho}(v, \rho) \delta \rho\right] d x d t+\int_{0}^{T} \int_{I} \psi_{\xi_{e}}\left(\xi_{e}\right) \delta \xi_{e} d x d t \\
& +\int_{0}^{T}\left(\int_{I} \mathcal{I}_{1} d x+\int_{I} \mathcal{I}_{2} d x+\int_{I} \mathcal{I}_{3} d x\right) d t-\int_{0}^{T}\left\langle\mathcal{G}, \delta \xi_{e}\right\rangle d t+\mathcal{I}_{4}+\mathcal{I}_{5} \\
& +\int_{0}^{T}\left\langle\rho^{-1}(\lambda+2 \mu) \Delta \mathcal{G}+\mathcal{A}_{\varepsilon}^{\prime \prime}(v) h^{2} \mathcal{G}+\mathcal{B}_{\varepsilon}^{\prime \prime}(v) h^{2} \mathcal{P}^{*} \mathcal{G}, \delta v\right\rangle d t
\end{aligned}
$$

From the existence of the solution of the conjugate problem (4.1), we finally have the expression for $\Delta \mathcal{J}$

$$
\begin{aligned}
\Delta \mathcal{J}\left(\xi_{e}\right) & =\int_{0}^{T} \int_{I} \psi_{\xi_{e}}\left(\xi_{e}\right) \delta \xi_{e} d x d t+\int_{0}^{T}\left(\int_{I} \mathcal{I}_{1} d x+\int_{I} \mathcal{I}_{2} d x+\int_{I} \mathcal{I}_{2} d x\right) d t \\
& -\int_{0}^{T}\left\langle\mathcal{G}, \delta \xi_{e}\right\rangle d t+\mathcal{I}_{4}+\mathcal{I}_{5}
\end{aligned}
$$

With the estimates of (3.11) and (3.12) fromtheorem 7 and the assumptions of theorem 9 we have for everything $t \in(0, T)$.

$$
\begin{aligned}
\left|\int_{0}^{T} \int_{I}\left(\mathcal{I}_{1}+\mathcal{I}_{2}\right) d x d t\right|= & \mid \int_{0}^{T} \int_{I}\left(\int _ { 0 } ^ { 1 } \left[\left(\ell_{v}(v+\tau \delta v, \rho+\tau \delta \rho)-\ell_{v}(v, \rho)\right) \delta v\right.\right. \\
& \left.\left.+\left(\ell_{\rho}(v+\tau \delta v, \rho+\tau \delta \rho)-\ell_{\rho}(v, \rho)\right) \delta \rho\right] d \tau\right) d x d t \mid \\
\leq & \int_{0}^{T} \int_{I} \int_{0}^{1}\left|\left(\ell_{v}(v+\tau \delta v, \rho+\tau \delta \rho)-\ell_{v}(v, \rho)\right)\right| \delta v \mid d \tau d x d t \\
& +\int_{0}^{T} \int_{I}^{1} \int_{0}^{1}\left|\left(\ell_{\rho}(v+\tau \delta v, \rho+\tau \delta \rho)-\ell_{\rho}(v, \rho)\right)\right||\delta \rho| d \tau d x d t \\
\leq & \eta_{3} \int_{0}^{T} \int_{I} \int_{0}^{1}(|v+\tau \delta v-v|+|\rho+\tau \delta \rho-\rho|)|\delta \rho| d \tau d x d t \\
& +\eta_{4} \int_{0}^{T} \int_{I}^{1} \int_{0}^{1}(|v+\tau \delta v-v|+|\rho+\tau \delta \rho-\rho|)|\delta v| d \tau d x d t \\
= & \eta_{3} \int_{0}^{T} \int_{I} \int_{0}^{1}(|\tau \delta v|+|\tau \delta \rho|)|\delta v| d \tau d x d t \\
& +\eta_{4} \int_{0}^{T} \int_{I}^{1} \int_{0}^{1}(|\tau \delta v|+|\tau \delta \rho|)|\delta \rho| d \tau d x d t \\
= & \eta_{5} \int_{0}^{T} \int_{I} \int_{0}^{1}(|\tau \delta v|+|\tau \delta \rho|)(|\delta v|+|\delta \rho|) d \tau d x d t \\
= & \frac{\eta_{5}}{2} \int_{0}^{T} \int_{I}(|\delta v|+|\delta \rho|)^{2} d x d t \\
\leq & \eta_{6} \int_{0}^{T}\left(\|\delta v\|_{L^{2}\left(I ; \mathbb{R}^{3}\right)}^{2}+\|\delta \rho\|_{W^{1,2}(I ; \mathbb{R})}^{2}\right) d t \\
\leq & \eta_{7}\left\|\delta \xi_{\xi_{e}}\right\|_{\mathbb{X}_{\xi_{e}}^{0}}^{2}(m e s I)^{1 / 2}=O\left(\left\|\delta \xi_{e}\right\|_{\mathbb{X}_{\xi e}^{0}}\right)
\end{aligned}
$$




$$
\begin{aligned}
\left|\int_{0}^{T} \int_{I} \mathcal{I}_{3} d x d t\right| & =\left|\int_{0}^{T} \int_{I} \int_{0}^{1}\left(\psi_{\xi_{e}}\left(\xi_{e}+\tau \delta \xi_{e}\right)-\psi_{\xi_{e}}\left(\xi_{e}\right)\right) \delta \xi_{e} d \tau d x d t\right| \\
& \leq \int_{0}^{T} \int_{I} \int_{0}^{1}\left|\psi_{\xi_{e}}\left(\xi_{e}+\tau \delta \xi_{e}\right)-\psi_{\xi_{e}}\left(\xi_{e}\right) \| \delta \xi_{e}\right| d \tau d x d t \\
& \leq \eta_{8} \int_{0}^{T} \int_{I} \int_{0}^{1}\left|\xi_{e}+\tau \delta \xi_{e}-\xi_{e} \| \delta \xi_{e}\right| d \tau d x d t \\
& \leq \eta_{8} \int_{0}^{T} \int_{I} \int_{0}^{1}\left|\tau \delta \xi_{e}\right|\left|\delta \xi_{e}\right| d \tau d x d t \\
& \leq \frac{\eta_{8}}{2} \int_{0}^{T} \int_{I}\left|\delta \xi_{e} \| \delta \xi_{e}\right| d x d t \\
& \leq \eta_{8}\left\|\delta \xi_{e}\right\|_{\mathbb{X}_{\xi_{e}}^{0}}^{2}(m e s I)^{1 / 2}=O\left(\left\|\delta \xi_{e}\right\|_{\mathbb{X}_{\xi_{e}}}\right)
\end{aligned}
$$

The other members $I_{4}$ and $I_{5}$ are evaluated in the same way. we finally get to:

$$
\Delta \mathcal{J}\left(\xi_{e}\right)=\int_{0}^{T}\left\langle\psi_{\xi_{e}}\left(\xi_{e}\right)+\mathcal{G}, \delta \xi_{e}\right\rangle d t+O\left(\left\|\delta \xi_{e}\right\|_{\mathbb{X}_{\xi_{e}}^{0}}\right.
$$

Therefore, the functional $\mathcal{J}\left(\xi_{e}\right)$ is Fréchet differentiable from $\xi_{e}$, and its gradient is given by the formula $\mathcal{J}^{\prime}\left(\xi_{e}\right)=\int_{0}^{T}\left(\psi_{\xi_{e}}\left(\xi_{e}\right)+\mathcal{G}\right) d t$

where $\mathcal{G}$ is a solution to the dual problem(4.5). Thus, the theorem is proved.

\section{Necessary and Sufficient Condition of Optimality}

We have proved in subsection 3.2 that the problem (3.1) admits an optimal triplet $\left(v^{*}, \rho^{*}, \xi_{e}^{*}\right) \in C\left((0, T) ; \mathbb{K}_{d i v}^{0}\right) \times C((0, T)$; $\left.W^{1,2}(I ; \mathbb{R})\right) \times \hat{\mathcal{U}}^{a d}$. In this section, we characterize optimal control by giving the necessary conditions of optimality of the first-order for a family of $\left(P_{k}\right)$ integrable functions. However, we first give the following lemma according to the optimal control theory.

Theorem 10 Let $\hat{\mathcal{U}}^{a d}$ be a set in space $L^{\frac{2 s}{s-1}}\left(I, \mathbb{R}^{3}\right)$. Suppose $\mathcal{J}\left(\xi_{e}\right)$ is differentiable. Then, for the optimality of the command $\xi_{e}^{*}=\xi_{e}^{*}(x, t) \in \hat{\mathcal{U}}^{\text {ad }}$ in the problem (3.2) and (2.8), it is necessary that the condition :

$$
\mathcal{J}^{\prime}\left(\xi_{e}^{*}\right)\left(\xi_{e}-\xi_{e}^{*}\right) \geq 0
$$

holds $\forall \xi_{e}(x, t) \in \hat{\mathcal{U}}^{\text {ad }}$, where $\mathcal{J}^{\prime}\left(\xi_{e}^{*}\right)$ represents the derivative of $\mathcal{J}\left(\xi_{e}^{*}\right)$ for $\xi_{e}=\xi_{e}^{*}$.

If $\mathcal{J}\left(\xi_{e}\right)$ is convex, then the condition (5.1) is sufficient for the optimality of the command $\xi_{e}^{*}(x, t)$.

Proof. Let $\xi_{e}^{*}(x, t)$ be the optimal control. $\forall \xi_{e} \in \hat{\mathcal{U}}^{\text {ad }}$ and $\varepsilon \in(0,1)$, we have :

$$
\mathcal{J}\left(\xi_{e}^{*}\right)=\mathcal{J}\left[(1-\varepsilon) \xi_{e}^{*}+\varepsilon \xi_{e}^{*}\right] \leq \mathcal{J}\left[(1-\varepsilon) \xi_{e}^{*}+\varepsilon \xi_{e}\right]
$$

From (5.2) we can deduce that :

$$
\frac{1}{\varepsilon}\left(\mathcal{J}\left(\xi_{e}^{*}+\varepsilon\left(\xi_{e}-\xi_{e}^{*}\right)\right)-\mathcal{J}\left(\xi_{e}^{*}\right)\right) \geq 0
$$

Therefore, if we go to the limit in (5.3), and we get (5.1). On the other hand, assume that $\mathcal{J}\left(\xi_{e}\right)$ is strictly convex, then $\varepsilon \in(0,1)$ and we can get:

$$
\mathcal{J}\left[(1-\varepsilon) \xi_{e}^{*}+\varepsilon \xi_{e}^{*}\right]<(1-\varepsilon) \mathcal{J}\left(\xi_{e}^{*}\right)+\varepsilon \mathcal{J}\left(\xi_{e}\right)
$$

From (5.4) we can deduce that :

$$
\frac{1}{\varepsilon}\left(\mathcal{J}\left(\xi_{e}^{*}+\varepsilon\left(\xi_{e}-\xi_{e}^{*}\right)\right)-\mathcal{J}\left(\xi_{e}^{*}\right)\right)<\mathcal{J}\left(\xi_{e}\right)-\mathcal{J}\left(\xi_{e}^{*}\right)
$$

If the limit of (5.5) exists we get :

$$
\begin{gathered}
\lim _{\varepsilon \rightarrow 0} \frac{1}{\varepsilon}\left(\mathcal{J}\left(\xi_{e}^{*}+\varepsilon\left(\xi_{e}-\xi_{e}^{*}\right)\right)-\mathcal{J}\left(\xi_{e}^{*}\right)\right)<\mathcal{J}\left(\xi_{e}\right)-\mathcal{J}\left(\xi_{e}^{*}\right) \\
0 \leq \mathcal{J}^{\prime}\left[\xi_{e}^{*}\right]\left(\xi_{e}-\xi_{e}^{*}\right)<\mathcal{J}\left(\xi_{e}\right)-\mathcal{J}\left(\xi_{e}^{*}\right), \quad \forall \xi_{e} \in \hat{\mathcal{U}}^{a d}
\end{gathered}
$$

Which concludes the proof of the theorem. 


\subsection{First Variation of Functionals}

Let $\hat{\mathcal{U}}^{a d}$ be a convex, closed and bounded set in the space $L^{\frac{2 s}{s-1}}\left(I, \mathbb{R}^{3}\right)$ and $\Delta \mathcal{J} \equiv \epsilon \delta \mathcal{J}+O(\epsilon)$ wich $\epsilon \in(0,1)$. $\delta \mathcal{J}$ is called the first functional variation $\mathcal{J}$. Let us recall that $: \delta \mathcal{J}\left(\xi_{e}, \vec{p}\right)=\left.\frac{d}{d \epsilon} \mathcal{J}\left(\xi_{e}+\epsilon \vec{p}\right)\right|_{\epsilon=0}$ where $\vec{p}$ belongs to a vector space $\mathbb{V}$.

Consider a family of functions $\left(P_{k}\right) \stackrel{\text { def }}{=}\left\{\psi^{(k)}\left(\xi_{e}\right)+\mathcal{G}^{(k)} \xi_{e}, \quad k=0, \cdots, p+q\right\}$, where $\mathcal{G}^{(k)}$ are solutions to the conjugated problem. We have

$$
\Delta \mathcal{J}^{(k)}=\int_{0}^{T}\left\langle\partial_{\xi_{e}}\left[\psi^{(k)}\left(\xi_{e}\right)+\mathcal{G}^{(k)} \xi_{e}\right], \xi_{e}^{\epsilon}-\xi_{e}\right\rangle d t
$$

The first variation $\delta \mathcal{J}^{(k)}\left(\xi_{e}\right)$ of the functional $\mathcal{J}^{(k)}\left(\xi_{e}\right)$ is determined as follows :

$$
\begin{gathered}
\delta \mathcal{J}^{(k)}\left(\xi_{e}\right)=\lim _{\epsilon \rightarrow 0} \frac{1}{\epsilon} \int_{0}^{T}\left\langle\partial_{\xi_{e}}\left[\psi^{(k)}\left(\xi_{e}\right)+\mathcal{G}^{k} \xi_{e}\right], \epsilon\left(\xi_{e}-\xi_{e}^{*}\right)\right\rangle d t+O(\epsilon) \\
\delta \mathcal{J}^{(k)}\left(\xi_{e}\right)=\int_{0}^{T}\left\langle\partial_{\xi_{e}}\left[\psi^{(k)}\left(\xi_{e}\right)+\mathcal{G}^{(k)} \xi_{e}\right],\left(\xi_{e}-\xi_{e}^{*}\right)\right\rangle d t+O(\epsilon)
\end{gathered}
$$

which $\xi_{e}^{\epsilon}-\xi_{e}=\epsilon\left(\xi_{e}-\xi_{e}^{*}\right)$

\subsection{Establishing the Necessary Optimality Conditions}

Let's designate by $\mathbb{V}$ a vector space of dimension $\operatorname{dim}(\mathbb{V})=p+q+1$ and $\mathcal{F}_{a}^{(k)}$ a functional variation family of $\mathbb{V}$ defined by

$$
\left\{\mathcal{F}_{a}^{(k)}\left(\xi_{e}, \mathcal{G}^{(k)}\right)\right\} \stackrel{\text { def }}{=}\left\{\left(\delta \mathcal{J}_{a}^{(0)}, \delta \mathcal{J}_{a}^{(1)}, \cdots, \delta \mathcal{J}_{a}^{(p+2)}, \cdots, \delta \mathcal{J}_{a}^{(p+q)}\right)\right\}=\mathscr{K}^{(p q)}
$$

of eligible direction $a=\left\{\gamma_{k}\left(\xi_{e}-\xi_{e}^{*}\right)\right\}$, where $\gamma_{k}$ are non-negative coefficients and

$$
\delta \mathcal{J}^{(k)}\left(\xi_{e}\right)=\int_{0}^{T}\left\langle\partial_{\xi_{e}}\left[\psi^{(k)}\left(\xi_{e}\right)+\mathcal{G}^{(k)} \xi_{e}\right], \gamma_{k}\left(\xi_{e}-\xi_{e}^{*}\right)\right\rangle d t
$$

Let's prove that the set $\mathscr{K}^{(p q)}$ is a convex cone in vector space $\mathbb{V}$.

(i). $\mathscr{K}^{(p q)} \neq \varnothing$. Indeed

$\left\{\mathcal{F}_{a}^{(k)}\left(\xi_{e}, \mathcal{G}^{(k)}\right)\right\}=\{0, \cdots, 0\}$ corresponds to the eligible direction $a_{k}=\left\{0\left(\xi_{e}^{(k)}-\xi_{e}^{*}\right)\right\}=\{0\} \in \mathbb{V}$, therefore $\{0\} \in \mathscr{K}^{(p q)}$

(ii). Let $y>0$. Let's show that $\left\{y \mathcal{F}_{a}^{(k)}\left(\xi_{e}, \mathcal{G}^{(k)}\right)\right\}_{k=0, \cdots p+q} \in \mathscr{K}^{(p q)}$

Let's define the family $\gamma_{k} \mathscr{K}^{(p q)}$ by $\gamma_{k} \mathscr{K}^{(p q)}=\left\{y \mathcal{F}_{a}^{(k)}\left(\xi_{e}, \mathcal{G}^{(k)}\right)\right\}=\left\{y \delta \mathcal{J}_{a}^{(0)}, \cdots, y \delta \mathcal{J}_{a}^{(p+q)}\right\}$

where $y \delta \mathcal{J}^{(k)}\left(\xi_{e}\right)=\int_{0}^{T}\left\langle\partial_{\xi_{e}}\left[\psi^{(k)}\left(\xi_{e}\right)+\mathcal{G}^{(k)} \xi_{e}\right], y \gamma_{k}\left(\xi_{e}^{(k)}-\xi_{e}^{*}\right)\right\rangle d t$ of direction $a_{k}=\left\{y \gamma_{k}\left(\xi_{e}-\xi_{e}^{*}\right)\right\}$.

Such a direction corresponds to the family $\left\{\mathcal{F}_{y a}^{(k)}\left(\xi_{e}, \mathcal{G}^{(k)}\right)\right\}_{k=0, \cdots p+q} \in \mathscr{K}^{(p q)}$. So, $\left\{\mathcal{F}_{y a}^{(k)}\left(\xi_{e}, \mathcal{G}^{(k)}\right)\right\}=\left\{y \mathcal{F}_{a}^{(k)}\left(\xi_{e}, \mathcal{G}^{(k)}\right)\right\}$. Hence $\left\{y \mathcal{F}_{a}^{(k)}\left(\xi_{e}, \mathcal{G}^{(k)}\right)\right\}_{k=0, \cdots p+q} \in \mathscr{K}^{(p q)}$ et $\mathscr{K}^{(p q)}$ is a cone.

(iii). Let's show now, that the cone $\mathscr{K}^{(p q)}$ is convex. Indeed if $\left\{\mathcal{F}_{a}^{(p+q-1)}\left(\xi_{e}, \mathcal{G}^{(p+q-1)}\right)\right\}$ and $\left\{\mathcal{F}_{a}^{(p+q)}\left(\xi_{e}, \mathcal{G}^{(p+q)}\right)\right\}$ are two elements of the space $\mathbb{V}$ belonging to $\mathscr{K}^{(p q)}$ with eligible directions $a_{p+q-1}=\left\{\gamma_{p+q-1}\left(\xi_{e}-\xi_{e}^{*}\right)\right\}$ and $a_{p+q}=\left\{\gamma_{p+q}\left(\xi_{e}-\xi_{e}^{*}\right)\right\}$. We have

$a_{p+q-1}+a_{p+q}=\left\{\left(\gamma_{p+q}+\gamma_{p+q-1}\right)\left(\xi_{e}-\xi_{e}^{*}\right)\right\}$, where $\xi_{e}=\xi_{e}^{(p+q-1)}-\epsilon\left(\xi_{e}^{(p+q-1)}-\xi_{e}^{(p+q)}\right)$ with $\epsilon \in(0,1)$.

The first variation becomes

$$
\begin{aligned}
\delta \mathcal{J}_{a_{p+q}+a_{p+q-1}}^{(k)}\left(\xi_{e}\right) & =\int_{0}^{T}\left\langle\partial_{\xi_{e}}\left[\psi^{(k)}\left(\xi_{e}\right)+\mathcal{G}^{(k)} \xi_{e}\right],\left(\gamma_{p+q}+\gamma_{p+q-1}\right)\left(\xi_{e}-\xi_{e}^{*}\right)\right\rangle d t \\
& =\int_{0}^{T}\left\langle\partial_{\xi_{e}}\left[\psi^{(k)}\left(\xi_{e}\right)+\mathcal{G}^{(k)} \xi_{e}\right],\left(\gamma_{p+q}+\gamma_{p+q-1}\right)\left(\xi_{e}^{p+q-1}\right.\right. \\
& \left.\left.-\epsilon\left(\xi_{e}^{p+q-1}-\xi_{e}^{p+q}\right)-\xi_{e}^{*}\right)\right\rangle d t \\
& =\int_{0}^{T}\left\langle\partial_{\xi_{e}}\left[\psi^{(k)}\left(\xi_{e}\right)+\mathcal{G}^{(k)} \xi_{e}\right], \gamma_{p+q-1}\left(\xi_{e}^{(p+q-1)}-\xi_{e}^{*}\right)\right\rangle d t \\
& +\int_{0}^{T}\left\langle\partial_{\xi_{e}}\left[\psi^{(k)}\left(\xi_{e}\right)+\mathcal{G}^{(k)} \xi_{e}\right], \gamma_{p+q}\left(\xi_{e}^{(p+q)}-\xi_{e}^{*}\right)\right\rangle d t \\
& =\delta \mathcal{J}_{a_{p+q}}^{(k)}\left(\xi_{e}\right)+\delta \mathcal{J}_{a_{p+q-1}}^{(k)}\left(\xi_{e}\right)
\end{aligned}
$$


From (5.8) we can deduce that

$$
\left\{\mathcal{F}_{a_{p+q}}^{(k)}\left(\xi_{e}, \mathcal{G}^{(k)}\right)\right\}+\left\{\mathcal{F}_{p+q-1}^{(k)}\left(\xi_{e}, \mathcal{G}^{(k)}\right)\right\}=\left\{\mathcal{F}_{a_{p+q}+a_{p+q-1}}^{(k)}\left(\xi_{e}, \mathcal{G}^{(k)}\right)\right\} \in \mathscr{K}^{(p q)}
$$

So, we can conclude that the set $\mathscr{K}^{(p q)}$ is a convex cone in vector space $\mathbb{V}$.

Definition 11 The contsraints at the point $\xi_{e}^{*}$, part of restrictions $\mathcal{J}^{(k)}\left(\xi_{e}^{*}\right) \leq 0$, for which $\mathcal{J}^{(k)}\left(\xi_{e}^{*}\right)=0$, are called active. Those for which $\mathcal{J}^{(k)}\left(\xi_{e}^{*}\right)<0$, are called inactive at $\xi_{e}^{*}$. For $\mathcal{J}^{(k)}\left(\xi_{e}^{*}\right)<0$, it's clear that for a small enough $\epsilon$, we will also have $\mathcal{J}^{(k)}\left(\xi_{e}^{*}+\epsilon \vec{p}\right) \leq 0$, but if $\mathcal{J}^{(k)}\left(\xi_{e}^{*}\right)=0$ for some indices $k$, it is not easy to find a vector $\xi_{e}^{*} \in L^{1}\left((0, T) ; L^{\frac{2 s}{s-1}}\left(I ; \mathbb{R}^{3}\right)\right)$, such that, for $\epsilon$ small enough, $\xi_{e}^{*}+\epsilon \vec{p}$ satisfies all the constraints in(3.2a). It is therefore necessary to impose additional conditions on the constraints called qualification conditions. It's with these conditions that we can make "variations" around a $\xi_{e}^{*}$ point to test its optimality. In the following we consider a $\mathscr{H}^{(p q)}$ hyperplane support to the $\mathscr{K}^{(p q)}$ cone such that the entire cone is located in one of the closed half spaces defined by the $\mathscr{H}^{(p q)}$ hyperplane (the hyperplane enjoying this property may not be unique). The equation of $\mathscr{H}^{(p q)}$ can be written as $\sum_{i=0}^{p} \varpi_{i} x^{i}=\digamma$ where $x^{0}, \cdots, x^{p}$ are current coordinates, $\varpi_{i}$ the coefficients of the equation of this hyperplane and $\digamma \in \mathbb{R}$. As the product of all the coefficients $\varpi_{i}$ by the same non-zero number does not modify the hyperplane $\mathscr{H}^{(p q)}$, we can consider, by changing the signs of all the numbers $\varpi_{i}$, that the $\mathscr{K}^{(p q)}$ cone is located in a half-space $\mathscr{H}_{-}^{(p q)}: \sum_{i=0}^{p} \varpi_{i} x^{i} \leq \digamma$

Either the cone $\mathscr{K}^{(p q)}=\left\{\mathcal{F}_{a}^{(k)}\right\}$ is built from the optimal command $\xi_{e}^{*}(x, t)$ and $\mathscr{H}_{-}^{(p q)}$ the half space of the hyperplane $\mathscr{H}^{(p q)}$ is defined by a functional $\mathscr{J}^{(k)}=\left(\mathscr{J}^{(0)}, \cdots, \mathscr{J}^{(p+q)} \in \mathbb{V}\right.$, we have the following assumptions:

$$
\begin{gathered}
\sum_{k=0}^{p+q}\left|\mathscr{J}^{(k)}\right|>0 \\
\left\langle\mathscr{J}^{(k)}, \varpi_{i}\right\rangle_{\mathbb{V}} \leq\left\langle\mathscr{J}^{(k)}, \mathcal{F}_{a}^{(k)}\right\rangle_{\mathbb{V}}, \forall \mathcal{F}_{a}^{(k)} \in \mathscr{K}^{(p q)}, \forall \varpi_{i} \in \mathscr{H}_{-}^{(p q)}
\end{gathered}
$$

If $\mathcal{J}^{(k)}$ is $\mathscr{C}$-differentiable for $k=0, \cdots, p+q$ at the point $\xi_{e}^{*}(x, t)$, and $\mathcal{J}^{(k)}, k=p+1, \cdots, p+q$, continuous in the neighbourhood of $\xi_{e}^{*}(x, t)$, so for $\mathscr{J}^{(k)} \neq 0$ we obtain

$$
\left\langle\sum_{k=0}^{p+q} \mathscr{J}^{(k)} \mathcal{J}^{(k)^{\prime}}\left(\xi_{e}^{*}\right), \xi_{e}-\xi_{e}^{*}\right\rangle \geqslant 0 \quad \text { et } \quad \mathscr{J}^{(k)} \mathcal{J}^{(k)}\left(\xi_{e}^{*}\right)=0, \quad \forall k=1, \cdots, p+q .
$$

Theorem 12 Let $\xi_{e}^{*}=\xi_{e}^{*}(x, t) \in \hat{\mathcal{U}}^{\text {ad }}$ be the optimal control of the functional (3.1) characterized by the following control system, the adjoint system and inequality

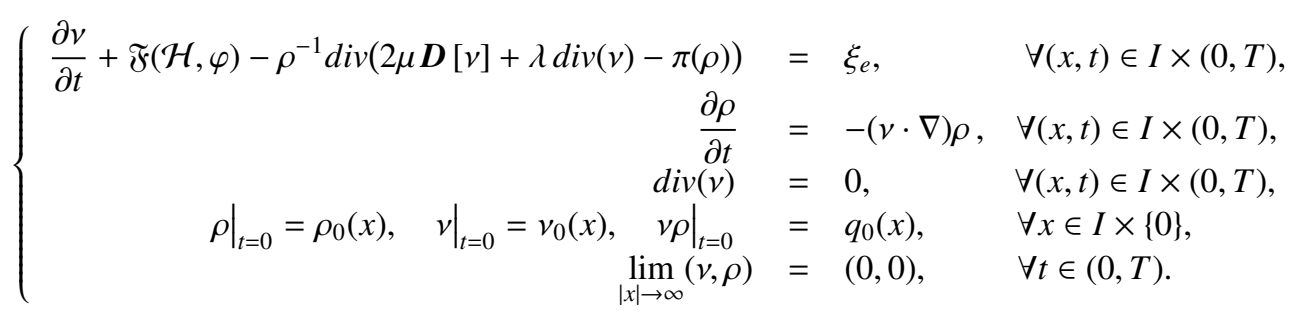

$$
\begin{aligned}
& \left\{\begin{array}{cc}
-\partial_{t} \mathcal{G}-\rho^{-1}(2 \mu+\lambda) \Delta \mathcal{G}+\left(\mathcal{A}_{\epsilon}^{\prime \prime}(v)+\mathcal{P}^{*} \mathcal{B}_{\epsilon}^{\prime \prime}(v)\right) \mathcal{G}-\nabla^{T} \rho \mathcal{Y} & =-\ell_{v}, \\
-\partial_{t} \mathcal{Y}-v \nabla \mathcal{Y} & =-\ell_{\rho}, \\
\xi_{e}(x, t) & =\mathcal{G}(x, t), \\
\sum_{j=1}^{3} \frac{\partial \mathcal{G}_{j}}{\partial x_{j}} & =0, \\
\left.\mathcal{G}\right|_{\partial I}=\left.\mathcal{Y}\right|_{\partial I} & =0, \\
\mathcal{G}(., T)=\mathcal{Y}(., T) & =0,
\end{array}\right.
\end{aligned}
$$

for any $(x, t) \in I \times(0, T)$.

$$
<\mathcal{J}^{\prime}\left(\xi_{e}^{*}\right), \xi_{e}-\xi_{e}^{*}>=\int_{0}^{T}\left\langle\partial_{\xi_{e}}\left[\psi^{(k)}\left(\xi_{e}\right)+\mathcal{G}^{(k)} \xi_{e}\right], \xi_{e}^{\epsilon}-\xi_{e}\right\rangle d t \geq 0
$$

$\forall \xi_{e}^{*}=\xi_{e}^{*}(x, t) \in \hat{\mathcal{U}}^{a d}$, where $\mathcal{G}^{(k)}$, solution of the conjugated problem (3.11) - (3.12). 
Proof. Let $\mathcal{F}_{a}^{(k)}$ be a family of functional variations of $\mathbb{V}$ defined by $\left\{\left(\delta \mathcal{J}_{a}^{(0)}, \cdots, \delta \mathcal{J}_{a}^{(p+q)}\right)\right\}$ of eligible direction $a=\left\{\gamma_{k}\left(\xi_{e}-\xi_{e}^{*}\right), \gamma_{k}>0\right\}$. Using the inequality(5.10), in which we stretch $\varpi_{i} \rightarrow 0$, we notice that $\delta \mathcal{J}_{a}^{(k)}$ satisfies (5.11) i.e.

$$
\forall \delta \mathcal{J}_{a}^{(k)} \in \mathscr{K}^{(p q)}, \quad 0 \leq \mathscr{J}^{(0)} \delta \mathcal{J}_{a}^{(0)}+\sum_{k=1}^{p} \mathscr{J}^{(k)} \delta \mathcal{J}_{a}^{(k)}+\sum_{k=p+1}^{p+q} \mathscr{J}^{(k)} \delta \mathcal{J}_{a}^{(k)}
$$

If $\mathscr{J}^{(k)}=0$, for $k=1, \cdots, p$, so for all inactive constraints $\mathcal{J}^{(k)}\left(\xi_{e}^{*}\right)<0$ and for active constraints $\mathcal{J}\left(\xi_{e}^{*}\right)^{k}=0$, we'll have

$$
\mathscr{J}^{(k)} \mathcal{J}_{a}^{(k)}\left(\xi_{e}^{*}\right)=0, \quad k=1, \cdots, p+q
$$

Let's introduce the following functions :

$$
\begin{aligned}
& \mathcal{Z}(x, t)=\sum_{k=0}^{p+q} \mathscr{J}^{(k)} \mathcal{G}(x, t) \\
& \mathscr{U}(x, t)=\sum_{k=0}^{p+q} \mathscr{J}^{(k)} \mathcal{Y}(x, t)
\end{aligned}
$$

with $(\mathcal{G}, \mathcal{Y})$ the problem solving couple (4.1). By multiplying (4.1) by $\mathscr{J}^{(k)}$ and using the formulas (5.15) and (5.16), the dual problem becomes :

$$
\left\{\begin{aligned}
-\partial_{t} \mathcal{Z}-\rho^{-1}(2 \mu+\lambda) \Delta \mathcal{Z}+\left(\mathcal{A}_{\epsilon}^{\prime \prime}(v)+\mathcal{P}^{*} \mathcal{B}_{\epsilon}^{\prime \prime}(v)\right) \mathcal{Z}-\nabla^{T} \rho \mathscr{U} & =-\sum_{k=0}^{p+q} \mathscr{J}^{(k)} \ell_{k v}, \\
-\partial_{t} \mathscr{U}-v \nabla \mathscr{U} & =-\sum_{k=0}^{p+q} \mathscr{J}^{(k)} \ell_{k \rho}, \\
\xi_{e}(x, t) \mathscr{J}^{(k)} & =\mathcal{Z}(x, t), \\
\sum_{j=1}^{3} \frac{\partial \mathcal{Z}_{j}}{\partial x_{j}} & =0, \\
\left.\mathcal{Z}\right|_{\partial I}=\left.\mathscr{U}\right|_{\partial I} & =0, \\
\mathcal{Z}(., T)=\mathscr{U}(., T) & =0 .
\end{aligned}\right.
$$

Thus, (5.14) can be transformed using the formula (5.15) again, and taking into account the fact that $\mathcal{F}_{a}^{(k)} \in \mathscr{K}^{(p q)}$ checks for inequality (5.13).

$$
\begin{gathered}
\mathscr{J}^{(0)}\left(\psi^{(0)}+\mathcal{G}^{(0)} \xi_{e}\right)+\sum_{k=1}^{p+q} \mathscr{J}^{(k)}\left(\psi^{(k)}+\mathcal{G}^{(k)} \xi_{e}\right) \geq 0, \\
\mathscr{J}^{(0)}\left(\psi^{(0)}+\mathcal{G}^{(0)} \xi_{e}\right)+\sum_{k=1}^{p+q} \mathscr{J}^{(k)} \psi^{(k)}+\mathcal{Z} \xi_{e} \geq 0 .
\end{gathered}
$$

So by replacing $\delta \mathcal{J}_{a}^{(k)}$ by her expression from (5.6), we can deduce (5.12)

$$
\int_{0}^{T} \int_{I} \partial_{\xi_{e}}\left[\psi^{(k)}\left(\xi_{e}\right)+\mathcal{G}^{(k)} \xi_{e}\right]\left(\xi_{e}-\xi_{e}^{*}\right) d x d t \geq 0, \quad \forall \xi_{e} \in \hat{\mathcal{U}}^{a d} .
$$

This completes the proof. If the control $\xi_{e}^{*} \in \hat{\mathcal{U}}^{a d}$ is the minimum point of the functional $\mathcal{J}\left(\xi_{e}\right)$, then the necessary condition for control has been obtained.

\section{Conclusion}

In this paper, we have studied an optimal control problem governed by nonlinear dynamic equations with dynamic viscosity and volume. We obtain the existence of an optimal solution to this control problem and establish the first-order necessary condition. Our results lay the foundation of numerical experiments of this optimal control problem. This issue will be worked on in the near future.

\section{References}

Adams, R. A. (1975). Sobolev Spaces Pure and Applied Mathematics, Academic Press, 65, New York, London.

Anderson, A. R. A., \& Gerlee, P. (2007). An evolutionary hybrid cellular automaton model of solid tumour growth, Journal of Theoretical Biology, 246(4), 583-603. 
Anderson, A. R. A., \& Enderling, H. (2006). Mathematical modelling of radiotherapy strategies for early breast cancer, Journal of Theoretical Biology, 241(1), 158-171.

Anderson, A. R. A., \& Chaplain, M. A. J. (1998). Continuous and discrete mathematical models of tumor-induced angiogenesis, Bulletin of Mathematical Biology, 60(5), 857-899.

Barbu, V. (1993). Analysis and control of nonlinear infinite dimensional systems, academic press, New York.

Brezis, H. (1993). Analyse fonctionnelle, edition Masson.

Brezis, H. (1999). Analyse fonctionnelle, Théorie et applications, Dunos, Paris.

Fattorini, H. O. (1996). Optimal control problem with state constrains for semilinear distributed parameter systems, J. Optim. Theory Appl. 88, pp.25-59.

Fursikov, A. V. (2000). Optimal control of distributed systems: Theory and applications, American Mathemtical Society, Rhode Island.

Girault, V., \& Raviart, P. A. (1986). Finite Element Methods for Navier-Stokes Equations, Springer-Verlag, Berlin, New York.

Gossan, D., Pascal, G., \& al. (2018). Existence, uniqueness and $\mathscr{C}$-differentiability of solutions in a non-linear model of cancerous tumor.

Hamilton, W. R. (1827). Theory of Systems of Rays.. Transactions of the Royal Irish Academy.

Jean, B. L. (2011). Estimation de paramétres d'un modéle de croissance et introduction d'un modéle spécifique aux gliomes de tout grade, https://tel.archives-ouvertes.fr

Kansal, A. R., \& Torquato, S. (2000). Simulated brain tumor growth dynamics using a three-dimensional cellular automaton, Journal of Theoretical Biology, 203(4), 367-382.

Lagrange, J. L. (1788). Mécanique Analytique. Paris: Desaint, 512 p.;in 8.; DCC. 4.403,1.

Lions, J. L., \& Magenes, E. (1972). Nonhomogeneous Boundary Value Problems and Applications, Vol. 1, SpringerVerlag, New York-Heidelberg-Berlin.

Li, Y. (1993). Optimal control theory for infinite dimensional systém Birkhauser.

Ouralcheva, O. A. N. (1973). Equations linéaires et quasi linéaires du type elliptique. Moscou. Naouka, P 576.

Pillis, L. G., \& Radunskaya, A. E. (2003). The dynamics of an optimally controlled tumor model: A case study, Mathematical and Computer Modelling, 37, 1221-1244.

Pontryagin, L. S., Boltyanskii, V. G., Gamkrelidze, R. V., \& Mishenko, E. F. (1969). Mathematical theory of optimal processes, M.: Nauka, (in Russian).

Raymond, J. P. (2013). Optimal control of partial differential équations, Université Paul Sabatier, Lecture Notes.

Robyn, P. A., \& Sean, M. (2004). A history of the study of solid tumour growth the contribution of mathematical modelling. In: Bulletin of Mathematical Biology, 66(5), p.1039-1091.

Silvia, F. (2014). Exact nonreflecting boundary conditions for exterior wave equation problems. https://doi.org/10.2298/PIM1410103F

Sritharan, S. S. (1998). Optimal Control of Viscous Flow, SIAM Frontiers in Applied Mathematics, Piladelphia, Society for Industrial and Applied Mathematics.

Swanson, K. R., \& Alvord, E. C. (2000). A quantitative model for differential motility of gliomas in grey and white matter, Cell Proliferation, 33(5), 317-329.

Trenoguine, V. (1985). Analyse Fonctionelle, Edition Mir, Moscow, (in French).

William, V. M. (1932). On a law of growth of Jensen's rat sarcoma, In : The American Journal of Cancer 16.4, p.841-846 (cf.p.8).

\section{Copyrights}

Copyright for this article is retained by the author(s), with first publication rights granted to the journal.

This is an open-access article distributed under the terms and conditions of the Creative Commons Attribution license (http://creativecommons.org/licenses/by/4.0/). 